\title{
Improved performance of ELMy H-modes at high density by plasma shaping in JET
}

\author{
G Saibene $^{1}$, R Sartori ${ }^{1}$, A Loarte ${ }^{1}$, D J Campbell ${ }^{1}$, P J Lomas L $^{2}$, V Parail $^{2}$, \\ K D Zastrow ${ }^{2}$, Y Andrew ${ }^{2}$, S Sharapov ${ }^{2}$, A Korotkov ${ }^{2}$, M Becoulet ${ }^{3}$, \\ G T A Huysmans ${ }^{3}$, H R Koslowski ${ }^{4}$, R Budny ${ }^{5}$, G D Conway ${ }^{6}$, J Stober $^{6}$, \\ W Suttrop ${ }^{6}$, A Kallenbach ${ }^{6}$, M von Hellermann ${ }^{7}$ and M Beurskens ${ }^{7}$ \\ ${ }^{1}$ EFDA Close Support Unit, c/o MPI fur Plasmaphysik, 2 Boltzmannstrasse, 85748 Garching, \\ Germany \\ 2 Association Euratom/UKAEA, Culham Science Centre, Abingdon OX14 3EA, UK \\ ${ }^{3}$ Association Euratom/CEA, Cadarache, F13108 St Paul-lez-Durance, France \\ ${ }^{4}$ Forschungszentrum Jülich GmbH, Institut für Plasmaphysik, EURATOM Association, \\ Trilateral Euregio Cluster, 52425 Jülich, Germany \\ 5 PPPL, Princeton University, PO Box 451, Princeton, NJ, USA \\ ${ }^{6}$ Association Euratom/IPP, MPI fur Plasmaphysik, 2 Boltzmannstrasse, Garching, Germany \\ ${ }^{7}$ FOM/Euraton Instituut v Plasmaphysica 'Rijnhuizen', Nieuwegein, Trilateral Euregio Cluster, \\ The Netherlands
}

Received 8 February 2002, in final form 10 June 2002

Published 27 August 2002

Online at stacks.iop.org/PPCF/44/1769

\begin{abstract}
We present the results of experiments in JET to study the effect of plasma shape on high density ELMy H-modes, with geometry of the magnetic boundary similar to that envisaged for the standard $Q=10$ operation in ITER. The experiments described are single lower null plasmas, with standard $q$ profile, neutral beam heating and gas fuelling, with average plasma triangularity $\delta$ calculated at the separatrix $\sim 0.45-0.5$ and elongation $\kappa \sim 1.75$. In agreement with the previous results obtained in JET and other divertor Tokamaks, the thermal energy confinement time and the maximum density achievable in steady state for a given confinement enhancement factor increase with $\delta$. The new experiments have confirmed and extended the earlier results, achieving a maximum line average density $n_{\mathrm{e}} \sim 1.1 n_{\mathrm{GR}}$ for $H_{98} \sim 0.96$. In this plasma configuration, at $2.5 \mathrm{MA} / 2.7 \mathrm{~T}\left(q_{95} \sim 2.8\right)$, a line average density $\sim 95 \% n_{\mathrm{GR}}$ with $H_{98}=1$ and $\beta_{\mathrm{N}} \sim 2$ are obtained, with plasma thermal stored energy content $W_{\text {th }}$ being approximately constant with increasing density, as long as the discharge maintains Type I ELMs, up to $n_{\text {ped }} \sim n_{\mathrm{GR}}$ (and $n_{\mathrm{e}} \sim 1.1 n_{\mathrm{GR}}$ ).

A change in the Type I ELMs behaviour is observed for pedestal densities $n_{\text {ped }} \gtrsim 70 \% n_{\mathrm{GR}}$, with their frequency decreasing with density (at constant $P_{\text {sep }}$ ), enhanced divertor $\mathrm{D}_{\alpha}$ emission and increased inter-ELM losses. We show that this change in the ELM character at high pedestal density is due to a change in transport and/or stability in the pedestal region, with the ELMs changing from Type I to mixed Type I and Type II. The similarity of these observations with those in the Type II ELM regime in ASDEX Upgrade and
\end{abstract}


with other small ELM regimes in DIII-D, JT-60U and Alcator C-MOD is discussed.

Finally, we present the first results of experiments by studying in more detail the effects of the plasma boundary geometry, in particular by investigating separately the effect of the upper and lower triangularity, at high average $\delta$. We show that the changes to the lower $\delta$ (or of the radial position of the X-point) affect the pedestal parameters, the size of ELM energy losses as well as the global energy confinement of the plasma.

\section{Introduction}

The ELMy H-mode regime is the reference scenario for the $Q=10$ inductive operation of ITER (Aymar et al 2001), as well as the basis for performance projections for the design of future plasma burning experiments (Meade et al 2001). The research effort on ELMy H-modes in present-day Tokamaks has focused on two aspects: the development of plasmas reaching simultaneously high density and high confinement and, more recently, the exploration of scenarios that combine the required plasma core properties with acceptable ELM energy losses.

For the first aspect, experiments in JET (Saibene et al 1999) and other Tokamaks (Kamada et al 1996, Osborne et al 2000a and Stober et al 2000) have shown that increasing the plasma triangularity $\delta$ increases the H-mode pedestal pressure $p_{\text {ped }}$ which, in turn, leads to higher thermal energy content $W_{\text {th }}$ of the plasma and confinement enhancement factor $H_{\mathrm{H}}$, for a given density. Experimentally in ELMy H-modes with Type I ELMs, the increase of $p_{\text {ped }}$ with triangularity is due to higher pedestal density $n_{\text {ped }}$ being obtained for the same pedestal temperature $T_{\text {ped }}$ and fixed main plasma parameters (plasma current $I_{\mathrm{p}}$, toroidal field $B_{\mathrm{t}}$, input power $P_{\text {in }}$ and external gas fuelling $\left.\Phi_{\text {gas }}\right)$. In other words, increased plasma $\delta$ results in higher densities at the pedestal and in the core, for a given pedestal temperature, and therefore leads to improved plasma energy content, compared to otherwise similar plasmas with lower $\delta$. The detailed comparison of core plasma performance between existing experiments and extrapolation to ITER, relies on the understanding of the relationship between pedestal parameters, in particular $T_{\text {ped }}$, and the resulting core gradients. Although different 'degrees' of profile resiliency may be required to model existing experiments (for instance, refer to Nordman et al (1999) and Ryter et al (2001)) that may lead to different extrapolations to ITER (ITER Physics Basis 1999), both experimental results and modelling clearly show that the increased pedestal stability resulting from plasma shaping is essential for obtaining the required performance of a plasma with conventional core profiles.

The improved trade-off between density and confinement obtained by plasma shaping is dependent on the pedestal parameters, in particular on maintaining a minimum pedestal temperature (pressure) and Type I ELMs (Sartori et al 2001b, 2002). Type I ELMs are fast relaxations of the pedestal pressure gradients causing the expulsion of particles and energy, that are ultimately transported to the divertor on very fast timescale $(\sim 100 \mu \mathrm{s})$, and may result in very high power densities being deposited onto the divertor plates $\left(\sim \mathrm{MW} \mathrm{m}^{-2}\right)$. Although this is not a problem for present day devices, the erosion rate of the divertor is of great concern for ITER, since it could limit the lifetime of the divertor target plates to an unacceptable low number of pulses (both for $C$ and $W$ components) (Federici et al 2001). Recent analysis of Type I ELM energy losses in present experiments (Loarte et al 2001a, 2002a) has highlighted a machine-independent correlation between the ELM energy losses $\Delta W_{\mathrm{ELM}}^{\mathrm{N}}$ (normalized to the pedestal energy content $\left.W_{\text {ped }}\right)$ and the local pedestal collisionality $v_{\text {ped }}^{*}$, with $\Delta W_{\mathrm{ELM}}^{\mathrm{N}}$ decreasing 
for increasing $v_{\text {ped }}^{*}$. If pedestal collisionality is the only quantity defining the ELM size, and SOL energy transport is similar for ITER and present day devices, then Type I ELMs in the ITER reference scenario would not be acceptable for divertor erosion (Loarte et al 2001b).

In fact, effort in experiments has been directed to the development of plasma scenarios that combine small ELMs to high core confinement and pedestal pressure, while maintaining the required energy and particle exhaust across the edge transport barrier (ETB). A plasma regime with $\mathrm{H}$-mode confinement but without ELMs was first established in Alcator C-Mod (Hutchinson et al 2001), with the enhanced D-alpha (EDA) regime. Unfortunately, attempts to reproduce EDA conditions on JET have not been successful so far (Loarte et al 2000). The first observation of a transition from Type I to small ELMs at high plasma confinement was reported by DIII-D back in 1990 (Ozeki et al 1990). In that experiment, the suppression of Type I ELMs was triggered by increasing the plasma elongation at constant main plasma parameters, in a highly shaped plasma with $q_{95} \sim 7$. The formal identification of these small ELMs as a new type of ELM (Type II ELMs) was put forward by the DIII-D team in 1990 (DIII-D Team 1990). More recently, small ELMs and high core confinement plasmas have been obtained with the 'grassy ELMs regime' of JT-60U (Kamada et al 2002), and the Type II ELMs of ASDEX Upgrade (Stober et al 2001a). In the case of JT-60U, the high plasma core performance is obtained in high triangularity plasmas, by a combination of an ETB with an internal transport barrier (ITB), while in ASDEX Upgrade the Type II regime is an extension at high shaping and density of a standard ELMy H-mode scenario. Mixed Type I and Type II ELMs have been also observed for the first time in high triangularity ELMy H-modes in JET, with high pedestal density and pressure (Loarte et al 2001a, Saibene et al 2001, Sartori et al 2001b and Becoulet et al 2002), and are analysed in detail in this paper. In all cases, as it will be discussed in section 3.3, the modification in the ELM behaviour appear to be correlated experimentally with high plasma shaping and magnetic shear in the edge pedestal region that, in turn, affects MHD stability and ELM size (Connor 1998).

This paper describes the results of experiments carried out in JET with the MkII gas box divertor to study the effect of plasma shaping, in particular triangularity, on both core and pedestal parameters of high density ELMy H-modes. The main experimental data originate from a series of density scans in high $\delta$ plasmas at $q_{95} \sim 3$, but density scans at higher $q_{95}$ and with reduced lower triangularity, compared to the reference set of data, are also discussed.

After a description of the experimental conditions (section 1.1) and of the general results of the experiments (section 2), the global confinement analysis is presented and compared with previous data from triangularity scans (section 2.1), while core profile effects are discussed in section 2.2. Section 3 describes the pedestal parameters and ELM behaviour in the density scans, focusing in particular on the characterization of the mixed Type I-II ELMs at high density. The effects of the variation of the lower plasma triangularity on ELMs and pedestal are discussed in section 3.2. The discussion section 3.3, examines similarities and differences of the ELM behaviour observed in JET at high $n_{\text {ped }}$ and high $\delta$, with small ELM regimes observed in other experiments and briefly discusses possible links between Type II ELMs and pedestal MHD stability. Finally, the results are summarized in section 4.

\subsection{Description of the experiments}

The plasmas studied are in lower single null configuration, with $D_{2}$ gas fuelling and $D$ neutral beam injection (NBI). Density scans were carried out by increasing the gas fuelling rate from pulse to pulse, keeping it constant for each discharge. The maximum fuelling rate was determined by either the Type I-III transition or by the technical limitation imposed by the maximum neutral pressure allowed in the NBI ducts. The divertor cryopump was at liquid $\mathrm{He}$ 


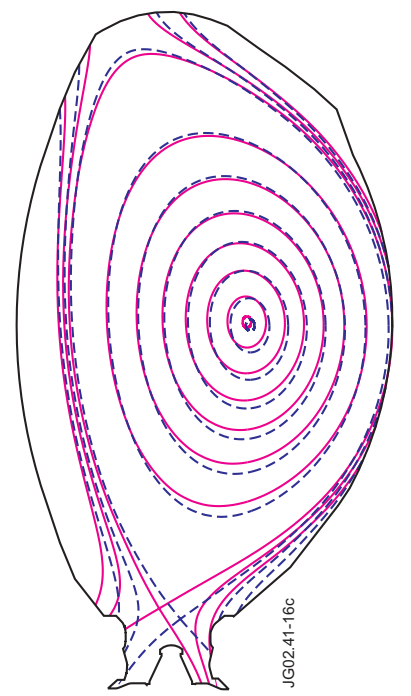

Figure 1. EFIT equilibrium reconstruction of pulse $52014\left(\delta \sim 0.47-\delta_{\text {up }} \sim 0.49, \delta_{\text {low }} \sim 0.45\right)$ in full line, and of pulse $52819\left(\delta \sim 0.40-\delta_{\text {up }} \sim 0.51, \delta_{\text {low }} \sim 0.30\right)$, dashed line.

temperature for all the experiments, and the average first wall temperature $T_{\text {wall }}$ was $\sim 320^{\circ} \mathrm{C}$. A subset of the scans was repeated with $T_{\text {wall }}$ lowered to $200^{\circ} \mathrm{C}$, to study the effect of wall recycling on the $\mathrm{H}$-mode density and on ELMs. The results of those experiments are reported in (Becoulet et al 2002), and are not further discussed here.

In the previous JET experiments on the effect of plasma $\delta$ on ELMy H-mode properties, reported in Saibene et al (1999), the maximum average $\delta$ (calculated at the separatrix) was $\sim 0.38$, with $\delta \equiv 0.5 \times\left(\delta_{\text {up }}+\delta_{\text {low }}\right)$, where $\delta_{\text {up } / \text { low }}$ are the upper and lower triangularity. To extend the range of previous data, new density scans were carried out at similar current and field as before $\left(2.5 \mathrm{MA} / 2.7 \mathrm{~T}, q_{95} \sim 2.8-3.1\right)$, but with the average $\delta$ increased to $0.47\left(\delta_{\text {up }} \sim 0.49\right.$ and $\delta_{\text {low }} \sim 0.45$ ), near to the value of $\delta \sim 0.5$ selected for ITER. The increase in triangularity corresponds to an increase in the magnetic shear in the edge region (as calculated with EFIT) from $\sim 4$ for $\delta \sim 0.38$ to $\sim 4.8$ for the plasmas with $\delta \sim 0.47$. For these scans, the input power was $P_{\text {in }} \sim 15 \mathrm{MW}$. The EFIT reconstruction of the equilibrium for one of the discharges in the series is shown in figure 1 , full line

The effect of $q_{95}$ on the pedestal and ELM behaviour was explored in density scans carried out with $P_{\text {in }}$ and plasma geometry as above; two values of $q_{95}$ of $\sim 3.8$ and $\sim 5$ were obtained by lowering $I_{\mathrm{p}}$ to $2.0 \mathrm{MA}$ and $1.5 \mathrm{MA}$, respectively. The effect of increasing $q_{95}$ at fixed plasma current was not studied, since the NBI power available in JET at the time was not sufficient to sustain an ELMy H-mode at high density and toroidal field (Sartori et al 2001b, 2002).

Finally, the role of plasma shape for pedestal and core properties of high density ELMy $\mathrm{H}$-mode has been further investigated by comparing the properties of discharges with similar $\delta_{\text {up }} \sim 0.5$ but different $\delta_{\text {low }}$, reduced from $\delta_{\text {low }} \sim 0.45$ to $\sim 0.30$ (see figure 1 , dashed line). The plasma current and field for the low $\delta_{\text {low }}$ discharges were $2.5 \mathrm{MA} / 2.7 \mathrm{~T}$, to match the reference set of density scans. 


\subsection{Diagnostic set-up}

The standard set of diagnostics for ELMy H-mode analysis, that requires simultaneous measurements of core and pedestal parameters, was available for all the plasma discharges studied in this paper. In particular, for the plasma core, $T_{\mathrm{e}}$ profiles were measured with ECE heterodyne radiometry (typically from $\rho \sim 0.4$ to $\sim 1, \rho=$ normalized minor radius) and with LIDAR, $T_{\mathrm{i}}$ profiles by active CXRS, and the electron density with a multi-chord FIR interferometer and LIDAR. Particular care was taken in the measurement of the pedestal $T_{\mathrm{e}}$, $T_{\mathrm{i}}$ and $n$. In particular, ECE provides fast $T_{\mathrm{e}}$ measurement, with typical time resolutions of $\sim 1 \mathrm{~ms}$ for several seconds and down to $4 \mu \mathrm{s}$ in selected time windows, with space resolution in the pedestal region $\sim 1-2 \mathrm{~cm}$. For best results, the plasma toroidal field $B_{\mathrm{t}}=2.7 \mathrm{~T}$ was selected for the main experiments to ensure good spatial resolution with the heterodyne system as well as to have a high value of the density cut-off for ECE emission $\left(\sim 8.5 \times 10^{19} \mathrm{~m}^{-3}\right.$ or $\sim 85 \%$ of the Greenwald limit $n_{\mathrm{GR}}$ at $2.5 \mathrm{MA}$, at the radius of the pedestal top). Nonetheless, at the highest densities for the $2.5 \mathrm{MA} \mathrm{scan}, n_{\mathrm{ped}} \sim n_{\mathrm{GR}}$ (maximum values at the end of the ELM cycle, figure 2), therefore, for these particular discharges, the measurement of the $T_{\mathrm{e} \text {,ped }}$ at the pedestal top is not available for the entire ELM cycle. In these cases, an estimate of $T_{\text {e,ped }}$ is obtained by using the value of $T_{\text {e,ped }}$ measured shortly after a Type I ELM crash, where $n_{\text {ped }}$ is at its minimum and just below the cut-off density for ECE (see section 3 ). Edge $T_{\mathrm{i}}$ profiles are measured with a dedicated edge CXRS system, providing data in the region $0.93 \lesssim \rho \lesssim 0.99$, with $\sim 2 \mathrm{~cm}$ and $50 \mathrm{~ms}$ resolutions. For the type of plasmas studied in this paper, $T_{\mathrm{i} \text {,ped }} \sim T_{\mathrm{e} \text {,ped }}$, therefore the pedestal analysis is carried out using $T_{\mathrm{e} \text {,ped }}$, to benefit from the high time resolution of this measurement, and the pedestal pressure $p_{\text {ped }} \propto n_{\mathrm{i}} T_{\mathrm{i}}+n_{\mathrm{e}} T_{\mathrm{e}}$ is approximated as $p_{\text {ped }} \propto 2 n_{\mathrm{e}} T_{\mathrm{e}}$.

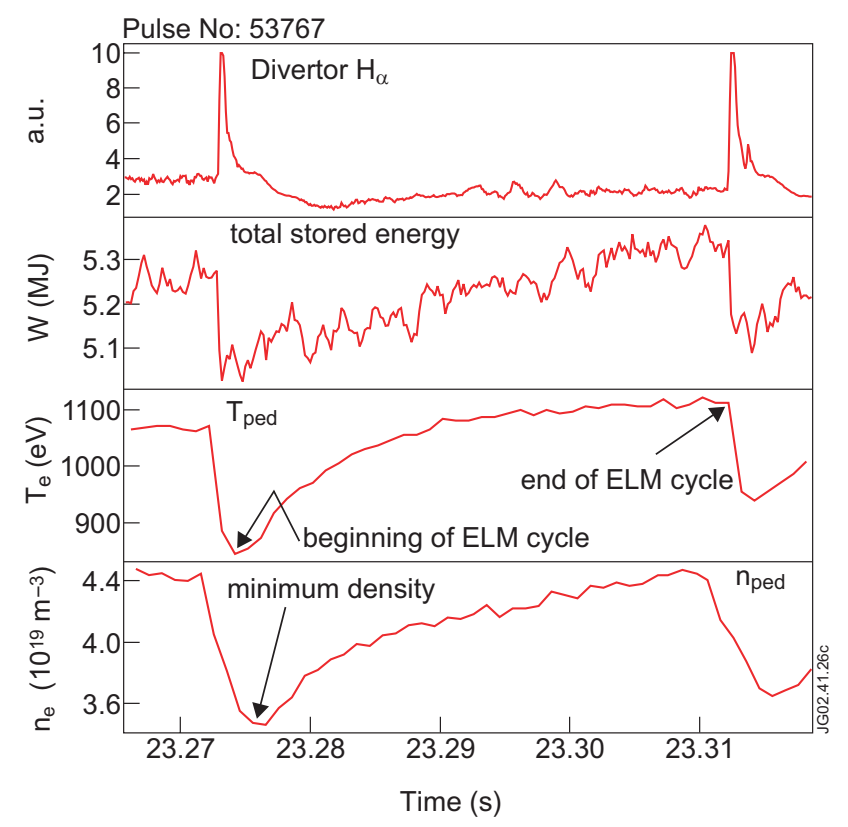

Figure 2. Typical time evolution of the plasma stored energy $W(\mathrm{MJ})$, pedestal temperature $T_{\text {ped }}$ $(\mathrm{eV})$ and density $n_{\text {ped }}\left(10^{19} \mathrm{~m}^{-3}\right)$ in a Type I ELMy H-mode. The 'ELM cycle' begins just after one ELM crash and ends just before the next ELM crash. Note that the pedestal density is at its minimum just after the crash. 
The density and temperature profiles in the pedestal region were measured with the edge LIDAR system (Beurskens et al 2001). Unfortunately, for most of the discharges at high $\delta$, the edge gradients scale length is shorter than the instrument spatial resolution, and the measured gradients saturate to a lower value than the real ones. Nonetheless, an estimate of the minimum pedestal pressure gradient $\nabla p_{\text {ped }}$ was obtained by combining edge LIDAR measurements of the $n_{\mathrm{e}}$ gradients with the $T_{\mathrm{e}}$ gradients determined by ECE, and used as an input for MHD stability calculations, as described in Becoulet et al (2002). Non-saturated measurement of edge density profiles were obtained with a Li Beam diagnostic (Brix et al 2001), although only for a few of the discharges analysed in this paper.

Fast (4 $\mu$ s time resolution) edge magnetic fluctuations measurements (Becoulet et al 2001), provided by an array of Mirnov coils, were available for most of the discharges, and used to characterize the changes in ELM behaviour observed at high density, whilst fast data (also with $4 \mu$ s time resolution) on density fluctuations, by fixed frequency reflectometry (Conway et al 1999), were available only for a restricted number of pulses (section 3.1.2).

\section{General results}

As outlined in section 1.1 , density scans were carried out at $2.5 \mathrm{MA} / 2.7 \mathrm{~T}$, at an average $\delta \sim 0.47$, increasing the gas fuelling rate from pulse to pulse. The time evolution of some plasma parameters and of the divertor $\mathrm{D}_{\alpha}$ emission are shown in figures 3 and 4 , for five selected discharges, in order of increasing $\Phi_{\text {gas }}$.

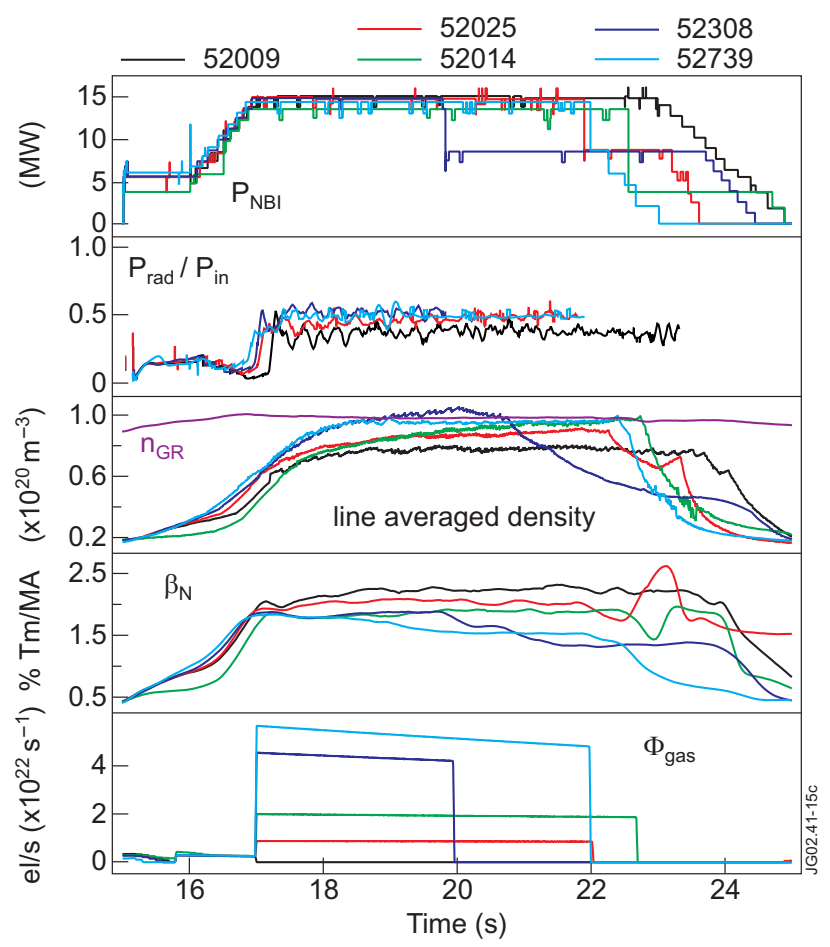

Figure 3. Time traces for five discharges in the density scans at $2.5 \mathrm{MA} / 2.7 \mathrm{~T}, \delta=0.47$. Boxes from top to bottom: NB power (MW), total radiated power fraction (100 ms smoothing applied), line average density $n_{\mathrm{e}}$ and $n_{\mathrm{GR}}\left(10^{20} \mathrm{~m}^{-3}\right), \beta_{\mathrm{N}}$ and last, gas fuelling rate $\Phi_{\text {gas }}\left(10^{22} \mathrm{~s}^{-1}\right)$. 


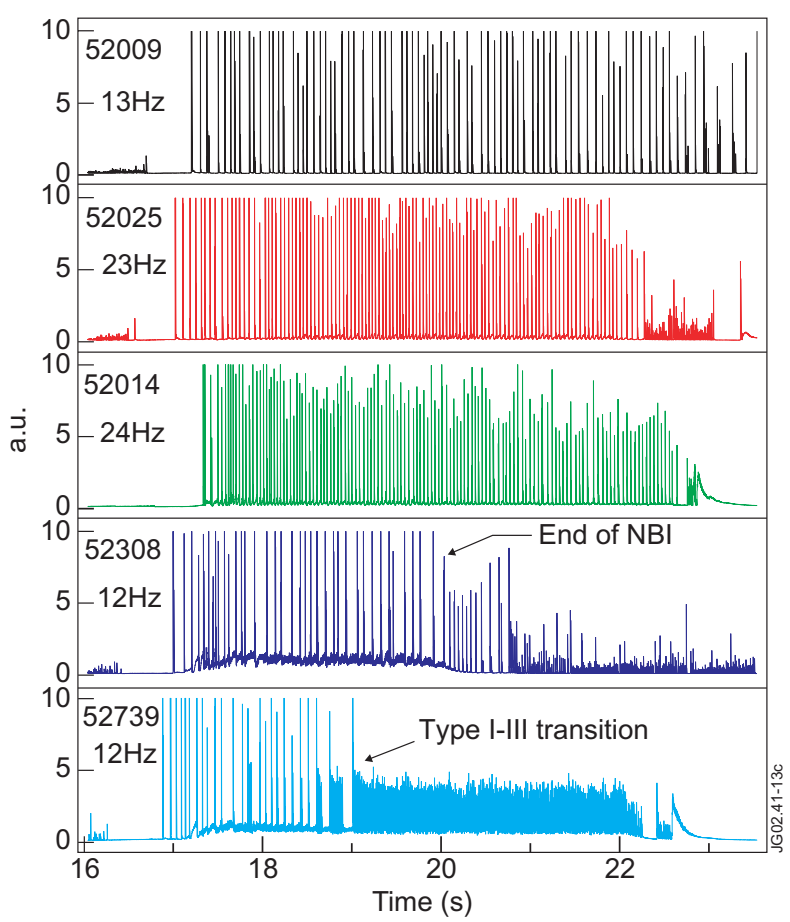

Figure 4. Time traces of the $\mathrm{D}_{\alpha}$ emission from the outer divertor region, for the same pulses as in figure 3 . The pulses are in order of increasing gas fuelling.

These experiments confirm and extend the earlier results on the positive effect of plasma triangularity to achieve high density at high confinement in ELMy H-modes. In fact, with $\delta=0.47$ at high fuelling rates $\left(\sim(3-4) \times 10^{22} \mathrm{~s}^{-1}\right)$ the plasma density obtained is $\gtrsim 90 \% n_{\mathrm{GR}}$ for several seconds with $\beta_{\mathrm{N}} \sim 2$. In these discharges, the total radiated power fraction increases with density from $\sim 35 \%$ to $\sim 55 \%$, with the change mainly due to increased divertor radiation.

The duration of the high density phase of these discharges is limited by the available NB pulse length to about $6 \mathrm{~s}$, corresponding approximately to $15 \tau_{\mathrm{E}}$. During the phase with constant $P_{\text {in }}$ and $\Phi_{\text {gas }}$, the plasma internal inductance $l_{\text {i }}$ slowly evolves, typically for $\sim 3 \mathrm{~s}$, and decreases by $5-10 \%$ to values of $0.85-0.9$. The final phase of the discharge, typically $\sim 2 \mathrm{~s}$ or $\sim 5 \tau_{\mathrm{E}}$ long, is then characterized by a constant value of $l_{\mathrm{i}}$.

The plasma density profile also shows a slow time evolution, as illustrated in figure 5, on similar timescales as $l_{\mathrm{i}}$. Initially, between 17 and $19 \mathrm{~s}$ in the discharge, the plasma density profile slowly peaks, although the fuelling is constant, and then peaking saturates, similarly to what is reported by Stober et al (2001b) for ASDEX Upgrade. The typical values of $n_{\text {peak }} \sim 1.1-1.4$, with the lower peaking observed at the higher density. Very peaked profiles are only measured after the Type I-III transition (pulse 52379 in figure 5, after 19 s), where the increase of $n_{\text {peak }}$ to $\sim 1.8$ is caused by the loss of pedestal density. Density profiles are discussed further in section 2.2.

\subsection{Global confinement}

The analysis of the typical timescale for the evolution of the main plasma parameters shows that the plasma discharges in the high $\delta$ density scan reach quasi-steady state after about $7 \tau_{\mathrm{E}}$. 


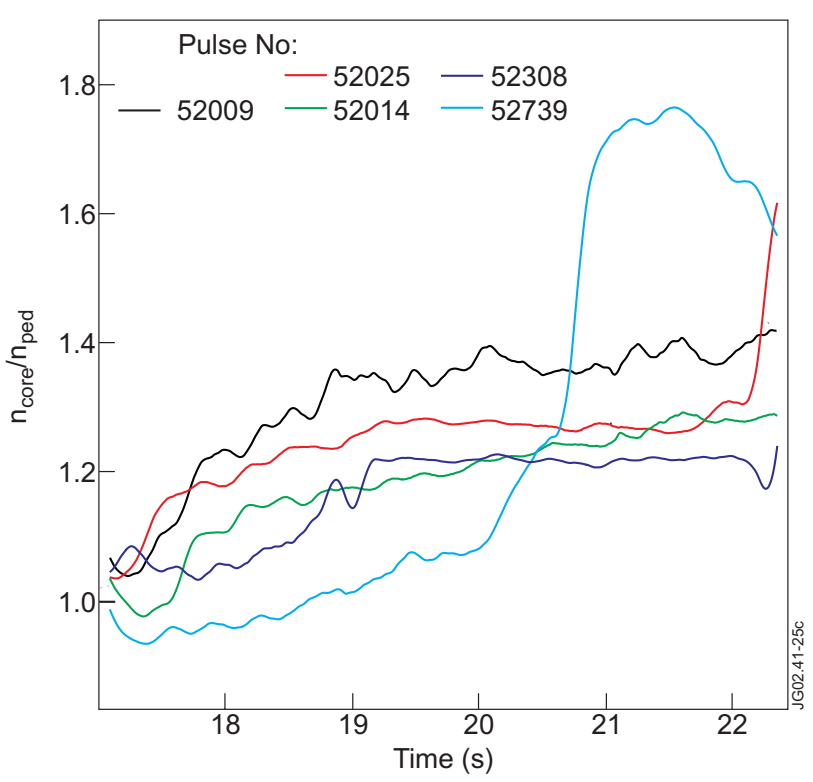

Figure 5. Time evolution of the density peaking factor $n_{\text {peak }}$ (calculated as the ratio of the central to the pedestal line average density, smoothed over $150 \mathrm{~ms}$ ), for the same set of pulses shown in figure 3 . The time window selected corresponds to the longest NB heating flat top.

Therefore, the calculation of the thermal stored energy $W_{\text {th }}$, and of the normalized confinement of these pulses (here the IPB98 ( $y$, 2) scaling is used (ITER Physics Basis 1999) has been carried out averaging late time slices in the pulse, and only one point per discharge is presented in the analysis. In a few cases, NB trips limited the pulse length (for example, pulse 52308 in figure 3 ), and the confinement point is taken at the end of the constant input power phase.

Figure 6 shows the $H_{98}$ confinement enhancement factor as function of density expressed as the Greenwald fraction $n_{\mathrm{e}} / n_{\mathrm{GR}}$, for the complete density scan at $2.5 \mathrm{MA} / 2.7 \mathrm{~T}, \delta \sim 0.47$ and $P_{\text {in }} \sim 15 \mathrm{MW}$. The increase of the plasma triangularity to $\delta=0.47$ results in a further improvement of the plasma thermal energy confinement obtained for a given plasma density. Nonetheless, also these data confirm the general observation that the energy confinement does not increase with density, as predicted by the scaling. Nonetheless, for $\delta \sim 0.47$, the maximum density achieved in Type I ELMs exceeds the Greenwald density limit (GDL) by $\sim 10 \%$, with confinement $H_{98} \sim 0.95$. For $n \sim 95 \% n_{\mathrm{GR}}$, the confinement enhancement factor of these plasmas is $H_{98}=1$, with $\beta_{\mathrm{N}}=2$ and $Z_{\mathrm{eff}}=1.4$. The combination of the normalized parameters achieved with the low $Z_{\text {eff }}$ and the edge $q_{95}=3$, matches or exceeds the specifications of global parameters for the $Q=10$ ELMy H-mode reference scenario of ITER (Aymar et al 2001).

More insight on shape effects can be gained from the analysis of the behaviour of the plasma thermal stored energy as a function of $n_{\text {ped }} / n_{\mathrm{GR}}$, shown in figure 7 for the same discharges of figure 6 . The absolute values of $W_{\text {th }}$ in figure 7 cannot be compared directly, since the NB input power was $\sim 14-15 \mathrm{MW}$ for the new data, whilst $P_{\text {in }} \sim 12 \mathrm{MW}$ in the old experiments. Nonetheless, figure 7 shows that, for each density scan at fixed $\delta, W_{\text {th }}$ is approximately constant as function of $n_{\mathrm{ped}} / n_{\mathrm{GR}}$ (and of $n / n_{\mathrm{GR}}$ as well, not shown), and that the degradation in energy content with density is typically $\lesssim 10 \%$ of the reference unfuelled value, as long as the plasma maintains Type I ELMs. With increasing $\delta$, the critical $n_{\text {ped }}$ for 
the Type I-III transition increases as well, whilst the critical temperature for the transition is similar (Sartori et al 2001a). The transition to Type III ELMs corresponds to a strong reduction of $W_{\text {th }}$ and of the pedestal density, particularly obvious for the scan at the highest triangularity (see figure 7, empty square), but observed in all cases.

\subsection{Core profile analysis}

Past JET results show that the pedestal pressure $p_{\text {ped }}$ is not constant for increasing $n_{\text {ped }}$, and $p_{\text {ped }} \propto n_{\text {ped }}^{-\alpha}$ with $\alpha \sim 1$ (Saibene et al 1999). A reduction of $p_{\text {ped }}$ for increasing $n_{\text {ped }}$ is also observed at $\delta=0.47$, although $p_{\text {ped }}$ stops decreasing at the onset of mixed Type I and Type II ELMs at high density (see section 3 ). Therefore, the observation that the plasma stored energy is approximately constant with $n_{\mathrm{e}}$ implies that the core confinement of these discharges is approximately constant or slightly increasing with density, since $W_{\text {ped }}$ decreases with density.

Based on this observation, we have analysed the changes in the core $n$ and $T$ profiles, for the $\delta=0.47,2.5 \mathrm{MA} / 2.7 \mathrm{~T}$ density scan. The density variation in the scan is large, from approximately 0.6 to $1.0 \times 10^{20} \mathrm{~m}^{-3}$ at the pedestal. Therefore, the central NB power and particle deposition profiles, calculated with the PENCIL code (Cox 1988), vary considerably, with the central power density and fuelling gradually decreasing (figure 8). In first approximation, if the core temperature profiles were self-similar, the combination of constant $W$ and off-axis heating should imply self-similarity in the density profiles or density peaking with density. In fact, as mentioned in section 2 , the density peaking factor decreases with density, down to $n_{\text {peak }} \sim 1.1$ for $n \sim n_{\mathrm{GR}}$ (figure 5). Therefore, the fact that $W_{\text {th }}$ is approximately constant with density is not due to density peaking compensating for the

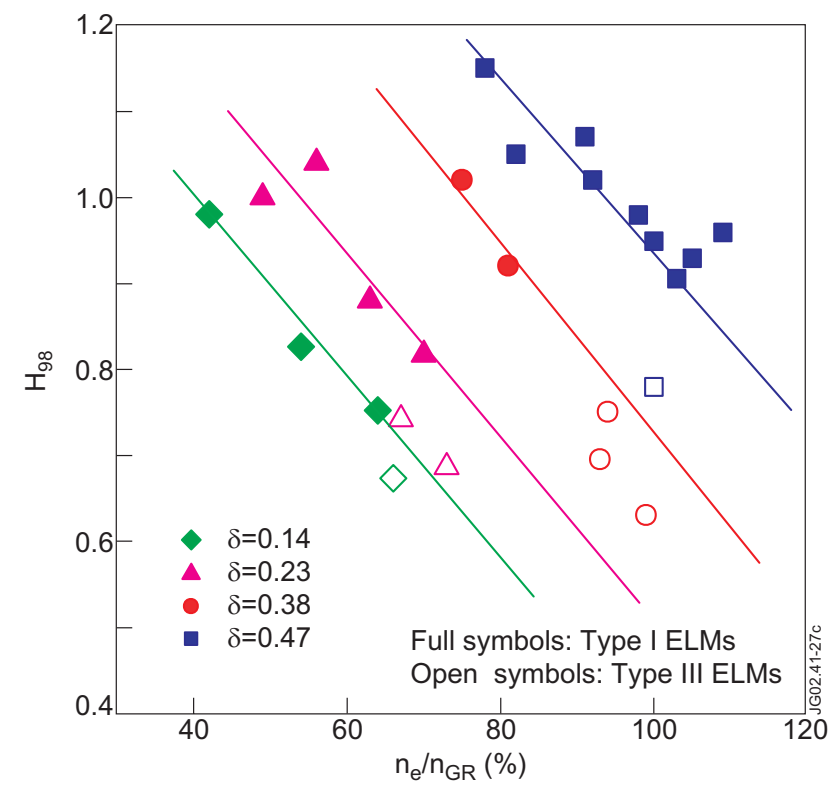

Figure 6. Confinement enhancement factor $H_{98}$ as function of the plasma line average density $n_{\mathrm{e}}$, normalized to the Greenwald density, $n_{\mathrm{GR}}$. The closed symbols are for data in Type I ELMy pulses, the open ones for Type III ELMs. The new data $(\delta=0.47$, squares, with GB to identify the MkII gas box divertor) are compared to those from the $\delta$ scans carried out in JET with the MkII divertor (Saibene et al 1999). 


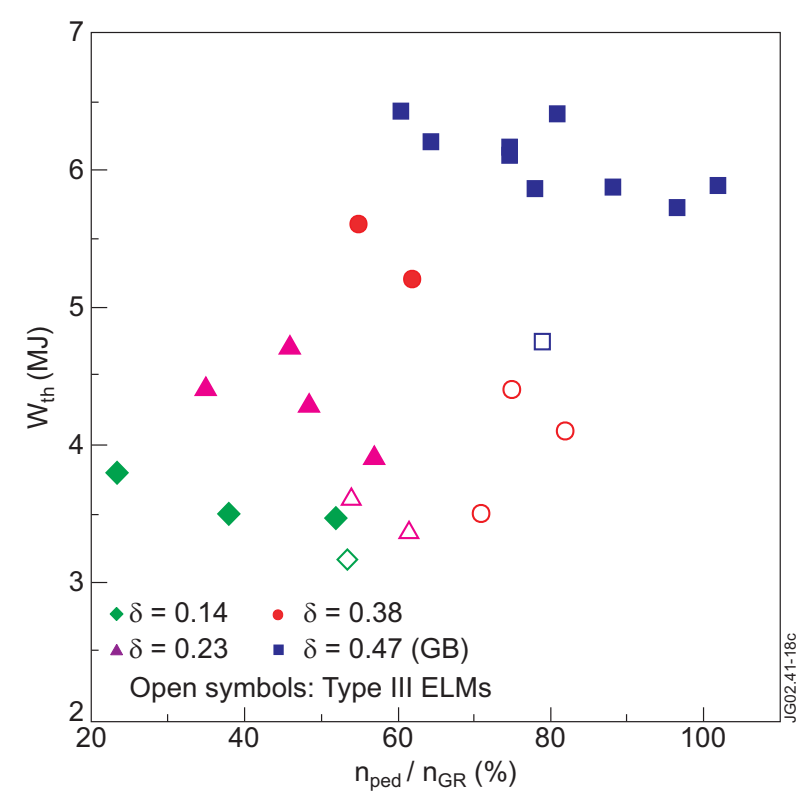

Figure 7. Thermal plasma stored energy $W_{\text {th }}$ as function of the pedestal density $n_{\text {ped }}$ normalized to the Greenwald density, for the same set of discharges as in figure 6 (same convention for the symbols). The non-thermal component of $W$ is calculated as described in Saibene et al (1999).

broadening of the power deposition profile (see Valovic et al (2002)) for a detailed discussion of the effects of input power on density profiles in JET). In particular, $T_{\mathrm{i}}$ and $T_{\mathrm{e}}$ are coupled in these discharges, due to the high density, and both decrease for increasing $n$, from $T_{\mathrm{i}} \sim 5 \mathrm{keV}$ and $T_{\mathrm{e}} \sim 4.5 \mathrm{keV}$ at $80 \% n_{\mathrm{GR}}$ to $\sim 2.2 \mathrm{keV}$ at $n \sim 1.1 n_{\mathrm{GR}}$. We find that the both $T_{\mathrm{e}}$ and $T_{\mathrm{i}}$ profiles are approximately self-similar up to $n \sim 80-85 \% n_{\mathrm{GR}}$ (figure 9). Beyond that density, we do not have information on the $T_{\mathrm{e}}$ profile behaviour, since the ECE emission is cut-off, but we observe that the $T_{\mathrm{i}}$ profiles become more peaked as the density increases. This observation is consistent with the reduction in $n_{\text {peak }}$ at constant $W_{\text {th }}$.

It is also interesting to note that slightly peaked density profiles are observed routinely in ELMy H-mode discharges in JET, and are not peculiar of high $\delta$ plasmas. For example, the density scan at $\delta=0.23$ (triangles in figures 6 and 7) has $n_{\text {peak }} \sim 1.4$ at low density, reduced to 1.3 at the highest density achieved in Type I ELMs in that particular series of discharges. Therefore, one can conclude that the improved confinement of high $\delta$ plasmas, at any density, is not due to increased density peaking with $\delta$, in contrast to the results reported by DIII-D (Osborne et al 2000b), where high confinement at density at or above $n_{\mathrm{GR}}$ was obtained by strong density peaking. On the other hand, triangularity affects the timescale for the density evolution, both for profiles and average values. For $\delta=0.23$, the typical timescale for the saturation of the density profile evolution is only $\sim 3 \tau_{\mathrm{E}}$, while it is almost twice as much for $\delta=0.47$. Also at high $\delta$, once the density profile shape is constant, we still observe a 'residual' time evolution of $n$ at constant $n_{\text {peak }}$ (compare figures 5 and 3), with very long characteristic times, so that in some cases the density does not saturate completely in the available NB pulse length. A similar effect is also found in lower $\delta$ discharges, but the density saturation time is shorter. One possible explanation of this difference with $\delta$ may come from the observation that ELM particle losses (normalized to $n_{\text {ped }}$ ) depend weakly on $\delta$ (Loarte et al 2001a, 2002a and Leonard et al 2001), whilst the ELM frequency at any density decreases with increasing $\delta$, for similar recycling conditions. Therefore, the particle outflux due to ELM losses is reduced 

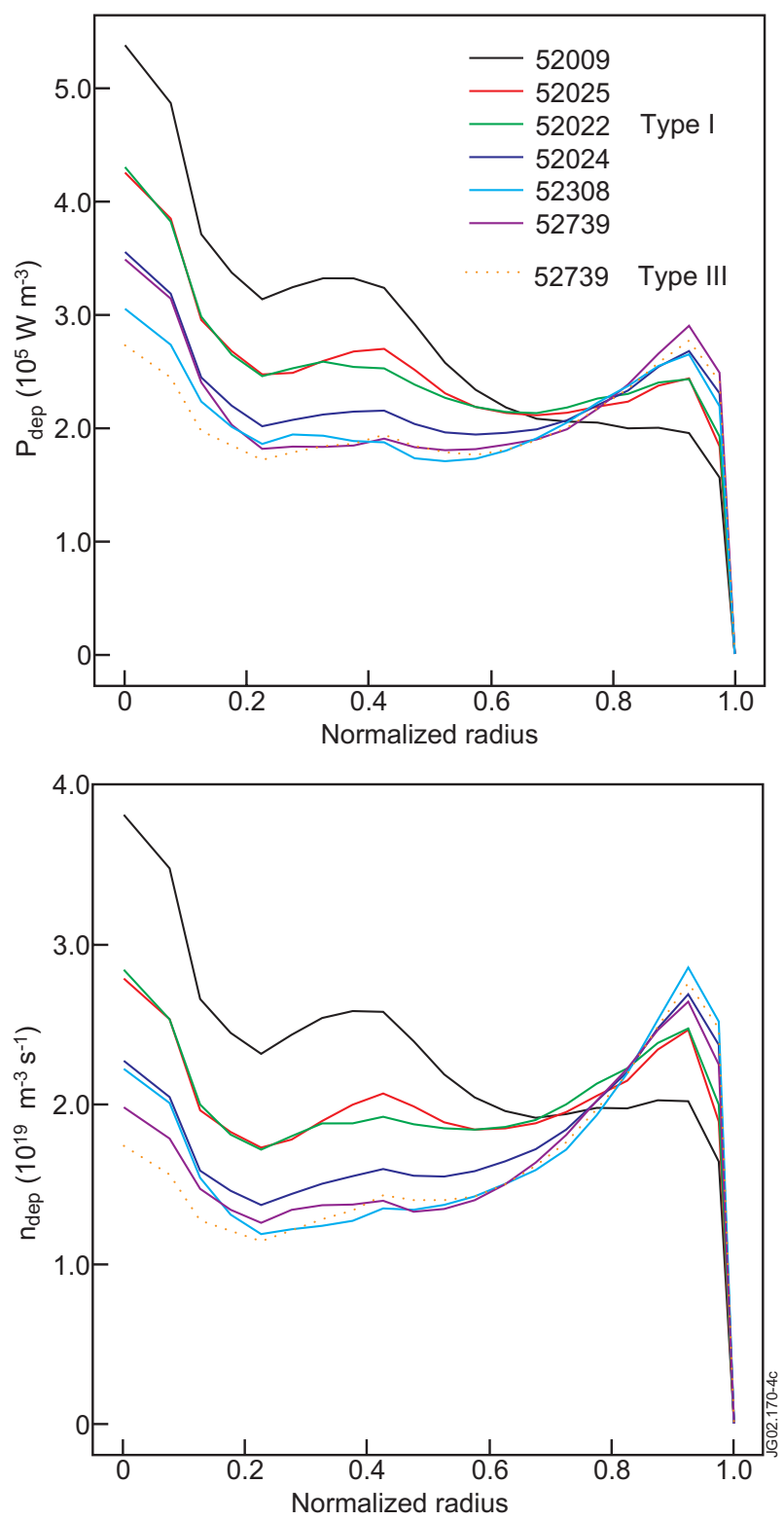

Figure 8. Flattening of the power density and particle deposition profiles for increasing density, for the same discharges as the $T_{\mathrm{i}}$ profiles in figure 9 . Each power and particle deposition profile is the average of several profiles in the discharge.

at high $\delta$, and density equilibration times may get longer because of this.

TRANSP analysis of the high $\delta$ density scan indicates a reduction of the effective heat conductivity $\chi_{\text {eff }}$ across the whole plasma radius for the discharges at the highest density (figure 10) although the heat flux is going down in the central part of the plasma but up in the periphery, consistent with the experimental observation on the $T_{\mathrm{i}}$ profiles.

This result may indicate that the assumptions of particle diffusion $D \propto \chi$ plus an inward particle pinch velocity $v_{\text {pinch }} \propto v_{\text {Ware }}$, successfully used to model ASDEX Upgrade plasmas 

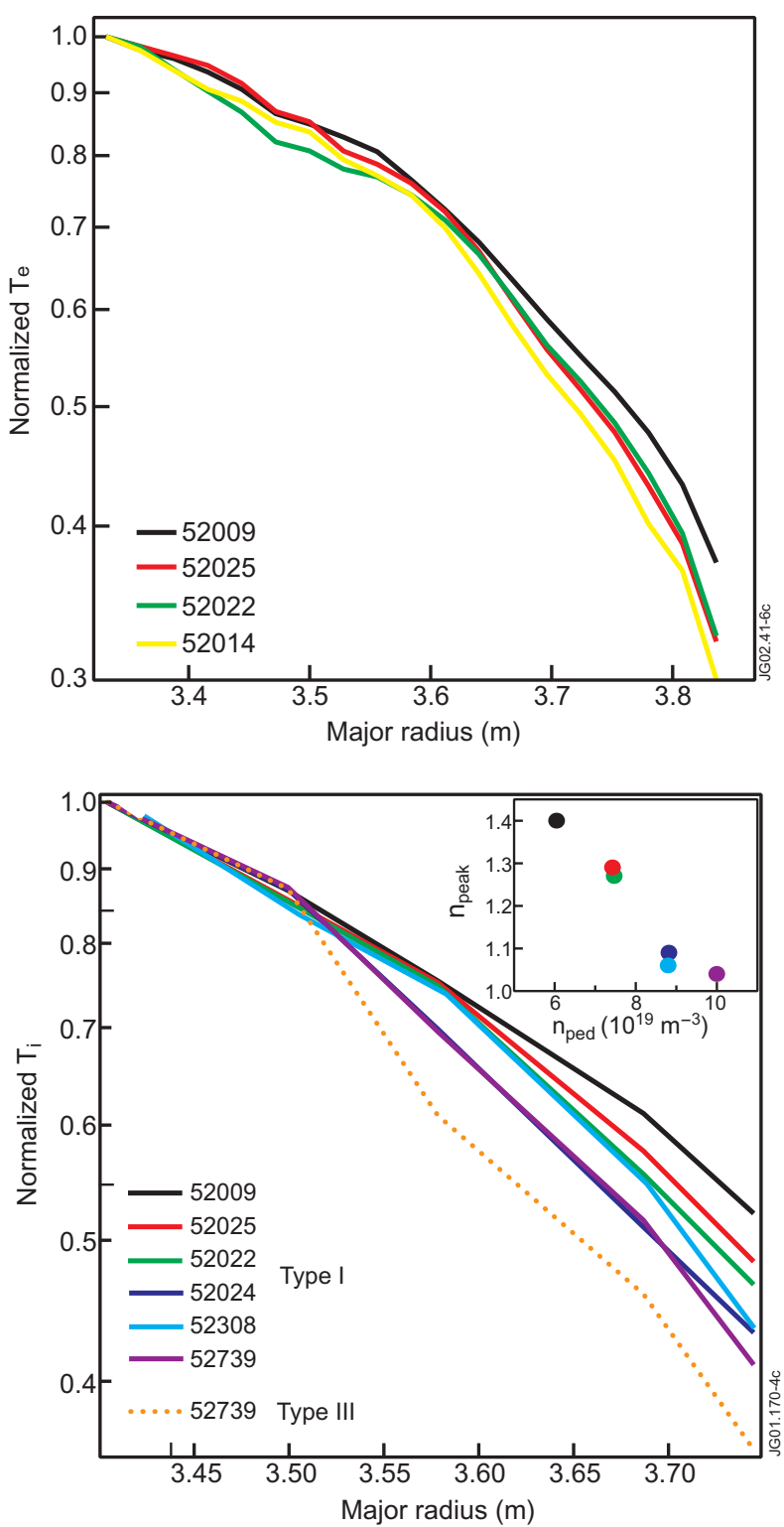

Figure 9. Main plasma $T_{\mathrm{e}}$ and $T_{\mathrm{i}}$ profiles for the a selection of pulses in the density scan at $\delta=0.47$ and 2.5 MA/2.7 T. Due to ECE emission cut-off, no $T_{\mathrm{e}}$ profiles are available for $n \gtrsim 0.85 \times 10^{20} \mathrm{~m}^{-3}$ (pulses 52024, 52308 and 52739). Each $T_{\mathrm{e}}$ profile is the average of three profiles, taken at times towards the end of the NB heating phase and just before a Type I ELM. For $T_{\mathrm{i}}$, each profile is also the average of three profiles, but not necessarily from the same ELM cycle. $T_{\mathrm{i}}$ profiles data for pulse 52014 are excluded for the plot because of below-standard quality. In both cases, the profiles are plotted on the region outside the sawtooth inversion radius. The inset in the plot of the $T_{\mathrm{i}}$ profiles shows the value of $n_{\text {peak }}$ for the same plasma discharges with Type I ELMs, for reference.

(Stober et al 2001b) not always applied to JET ELMy H-modes, as found in the modelling by Parail et al (2001) of JET plasmas with strong density peaking, since the reduced $\chi$ at high density is not associated to increased density peaking (i.e. to a reduced $D$ ). 


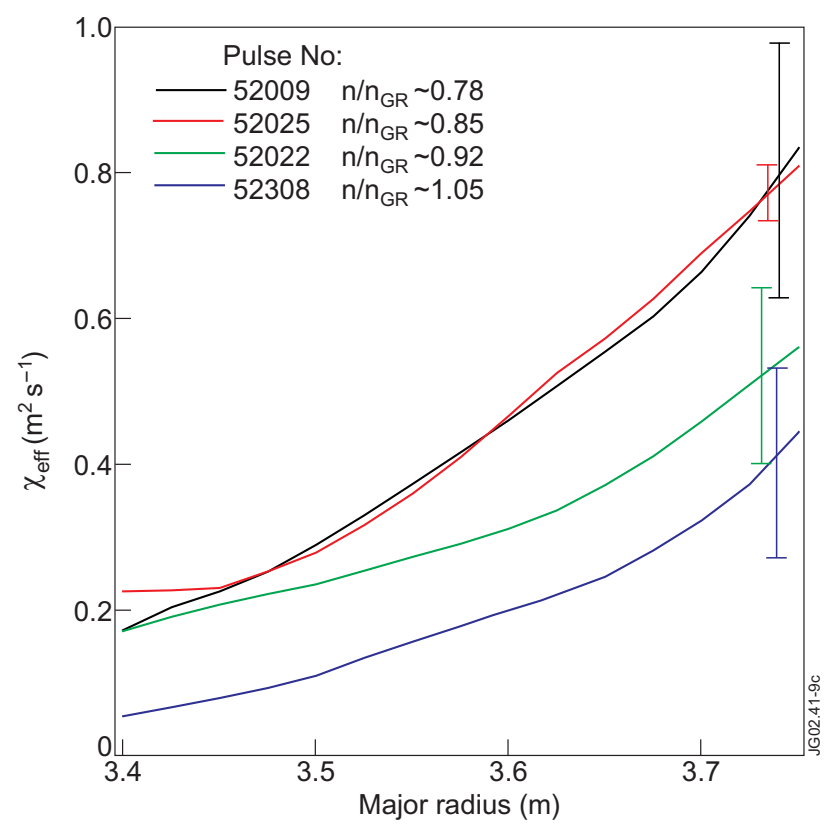

Figure 10. Time averaged $\chi_{\text {eff }}$ profiles for four discharges with increasing density, for the $\delta=0.47$ at $2.5 \mathrm{MA} / 2.7 \mathrm{~T}$ density scan.

\section{Pedestal and ELMs}

Figure 4 shows the time traces of the divertor $\mathrm{D}_{\alpha}$ signals for five representative discharges of the density scan at $\delta=0.47$ and $2.5 \mathrm{MA} / 2.7 \mathrm{~T}$. The inspection of the these traces shows an anomaly in the ELM frequency $f_{\mathrm{ELM}}$, since the usual relationship between Type I ELM frequency and pedestal density ( $f_{\mathrm{ELM}}$ increases with $\left.n_{\text {ped }}\right)$ breaks down at high density. In fact, for the first discharges in the density scan, with relatively low $n_{\text {ped }}\left(\lesssim 70 \% n_{\mathrm{GR}}\right.$ or $\left.7 \times 10^{19} \mathrm{~m}^{-3}\right)$, the Type I ELM frequency increases with density (and gas fuelling) as normal but, for $n_{\text {ped }} \gtrsim 70 \% n_{\mathrm{GR}}$, $f_{\mathrm{ELM}}$ stops increasing and then, for $n_{\mathrm{ped}} \sim 80 \% n_{\mathrm{GR}}, f_{\mathrm{ELM}}$ decreases, down to values similar or lower of those of the unfuelled reference pulse. A further small increase of the external gas fuelling (pulse 52739, figure 4) causes a transition to Type III ELMs.

The relationship between pedestal density and temperature also changes when the $f_{\mathrm{ELM}}$ anomaly occurs. As already mentioned in section 2.2 , in general $p_{\text {ped }}$ is not constant for increasing $n_{\text {ped }}$ in Type I ELMy H-modes in JET, and it scales approximately as $n^{-1}$. The $n_{\mathrm{e}}-T_{\mathrm{e}}$ edge diagram for the density scan at $\delta=0.47$ is shown in figure 11 , black dots. The plot shows clearly three features: first, for $n_{\text {ped }} \lesssim(7-8) \times 10^{19} \mathrm{~m}^{-3}\left(\sim 70-80 \% n_{\mathrm{GR}}\right), p_{\text {ped }}$ is decreasing with density as expected. Second, the reduction of $f_{\mathrm{ELM}}$ for $n_{\text {ped }} \gtrsim 8 \times 10^{19} \mathrm{~m}^{-3}$ corresponds to a change in the trend of $T_{\text {ped }}$, which stops decreasing with $n_{\text {ped }}$ and, as a consequence, we observe $p_{\text {ped }}$ now increasing with density, although not back up to the initial values of the unfuelled discharge. Note that in this density range, the temperature at the pedestal is taken at the beginning of the ELM cycle (see section 1.2), and therefore it represent a lower limit for the temperature of the pedestal at the end of the ELM cycle (where the density value is taken). Third, the $f_{\mathrm{ELM}}$ anomaly occurs at values of $T_{\text {ped }}$ just above the critical temperature for transition to Type III ELMs (see also Sartori et al (2001b)). This particular behaviour of the ELM frequency and of the pedestal pressure, first identified in JET for the discharges 


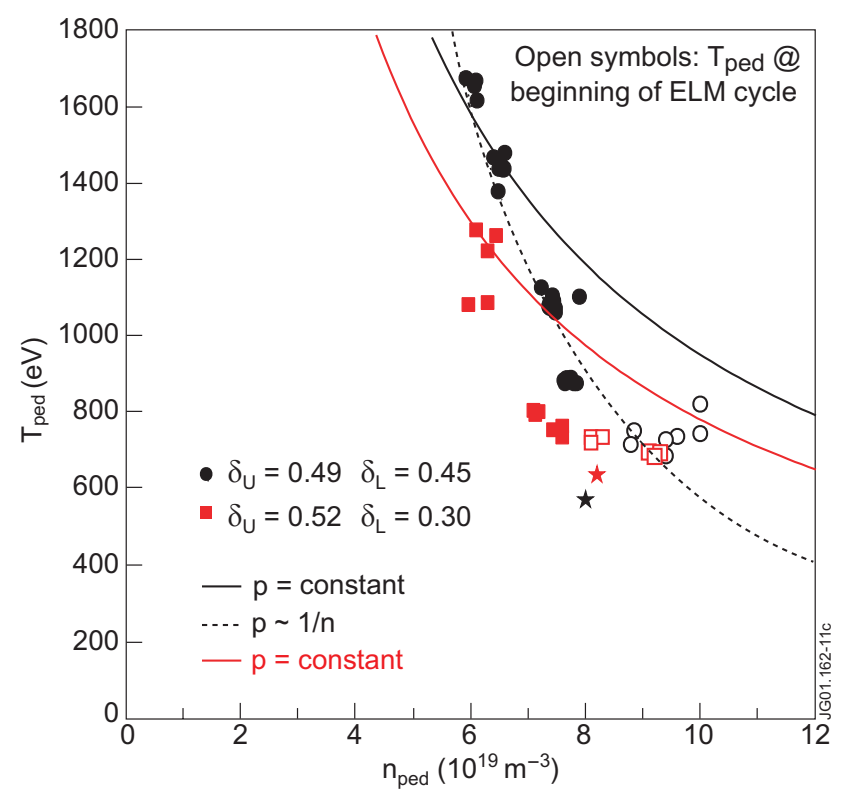

Figure 11. The $n_{\mathrm{e}}-T_{\mathrm{e}}$ edge pedestal diagram for two density scans. The black circles are for $\delta=0.47\left(\delta_{\text {low }}=0.45\right)$ at $2.5 \mathrm{MA} / 2.7 \mathrm{~T}$; the red squares are for the discharges at reduced $\delta_{\text {low }}=0.30$. For both sets of data, the open symbols are $T_{\text {ped }}$ values taken at the beginning of the ELM cycle, when the ECE emission is not cut-off, while $n_{\text {ped }}$ is taken at its maximum. The stars are for Type III ELMs. Also shown are the lines of constant $p_{\text {ped }}$ for both scans and the line $p \propto n^{-1}$ line for the high $\delta_{\text {low }}$ scan only.

described above, has been then observed in other JET plasmas. In the next section, we show that the reduction of the Type I ELM frequency at high pedestal pressure results from a change in the pedestal stability and/or edge transport. In analogy with the phenomenology identified in ASDEX Upgrade (Stober et al 2001a), we propose to identify this new phenomenon for JET as Type II ELMs, and show that in the JET plasmas, Type I and Type II ELMs may coexist at high pedestal density.

\subsection{Identification of Type II ELMs in JET}

The observed reduction of $f_{\mathrm{ELM}}$ with density is accompanied by a characteristic change of the divertor $\mathrm{D}_{\alpha}$ signal measured between Type I ELMs. Figure 12 shows a clear example of this, comparing pulses 52009 and 52308. The $\mathrm{D}_{\alpha}$ trace of 52308 shows an enhanced level of the base-line emission, compared to pulse 52009, with characteristic small and irregular oscillations between the Type I ELMs.

Several observations, which will be presented, indicate that the small oscillations between Type I ELMs are not Type III ELMs, and allow to conclude that what we observe are mixed Type I and Type II ELMs, as discussed below.

First, high density transitions from Type I to Type III ELMs in JET are always associated to a reduction of the plasma stored energy, pedestal pressure, and often also to a reduction of the pedestal density (Saibene et al 1998). The data in figures 7 (squares) and 11 (black squares) show, in contrast, that $W_{\text {th }}$ is constant and both $p_{\text {ped }}$ and $n_{\text {ped }}$ are near to the Type I ELM values, and increasing. This observation is consistent with measurements in mixed Type I-II regimes in both ASDEX Upgrade (Stober et al 2001a) and JT-60U (Kamada and 


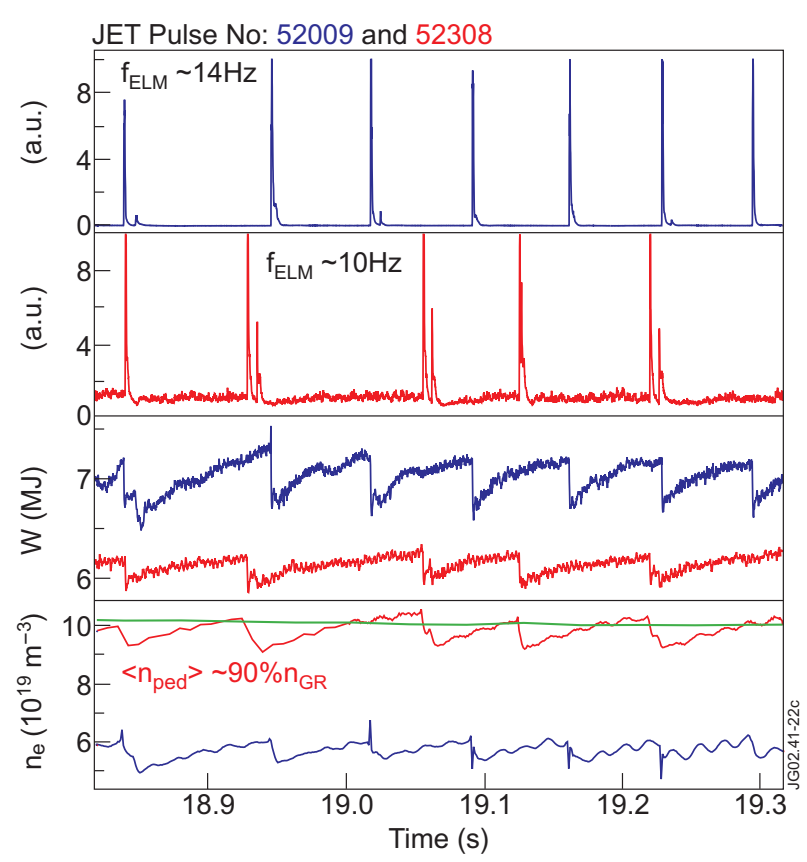

Figure 12. Comparison of the divertor $\mathrm{D}_{\alpha}$ traces of pulses 52009 and 52308 (box 1 and 2), with their respective total plasma stored energies $W$ (box 3 , values not corrected for fast ion content) and line average densities $n_{\mathrm{e}}$ (box 4).

the JT-60U Team 2001), figure 13, showing that the transition from Type I to Type II ELMs occurs at approximately constant $n_{\text {ped }}$ and $T_{\text {ped }}$. The behaviour of the pedestal pressure and density gradients also sets apart Type III ELMs and the small high frequency oscillations that we identify as Type II ELMs. Figure 14 shows the electron pressure profiles derived from edge LIDAR measurements, for discharges at different densities, and $\delta=0.47,2.5 \mathrm{MA} / 2.7 \mathrm{~T}$. As discussed in section 1.2, the space resolution of edge LIDAR diagnostic for the $2.5 \mathrm{MA}$ high $\delta$ pulses with Type I ELMs is not sufficient to resolve the pedestal gradients, and one can only say that $\nabla p_{\mathrm{e}}$ in the pedestal region is always at least $240 \mathrm{kPa} \mathrm{m}^{-1}$, and any change of the gradient with density is not resolved (Beurskens et al 2001). In contrast, $\nabla p_{\mathrm{e}}$ is resolved in the Type III ELMs phase, such as in pulse 52739 after $19 \mathrm{~s}$ (see figure 4). The transition to Type III ELMs corresponds to a reduction of $\nabla p_{\mathrm{e}}$ down to $\sim 180 \mathrm{kPa} \mathrm{m}^{-1}$, indicating indirectly that the edge pressure gradient sustained during mixed Type I and Type II ELM phases is substantially higher than with Type III ELMs.

The identification of a specific and unique MHD signature associated with this change in the ELM behaviour at high density gives a second argument for the identification of the interELM activity as Type II ELM phases. In fact, we observe a characteristic change of the MHD fluctuations between ELMs that display the $f_{\mathrm{ELM}}$ 'anomaly', with an enhancement of MHD fluctuations in the $10-30 \mathrm{kHz}$ region, in a very similar fashion to that reported by Stober et al (2001a) for ASDEX Upgrade. MHD and density fluctuations are discussed in more detail in section 3.1.2.

In summary, the change in the Type I ELM behaviour at high $\delta$ in JET is associated with broadband MHD activity, high $n_{\text {ped }}$ and $p_{\text {ped }}$, with pedestal temperatures marginally above the critical value for the Type I-III transition, and to good global energy confinement. These 


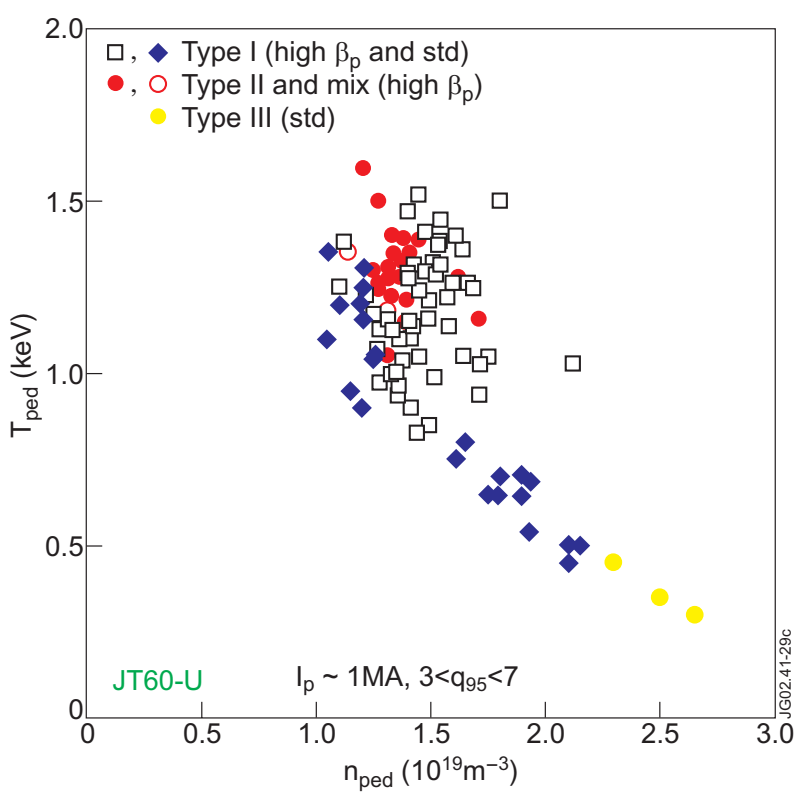

Figure 13. The $n_{\mathrm{e}}-T_{\mathrm{e}}$ diagram for a series of discharges from JT-60U (courtesy of Y Kamada, JAERI). The diagram includes the values of the electron temperature and density at the top of the pedestal for Type I ELMy H-modes (labelled std, standard, in the figure), high $\beta_{\mathrm{p}} \mathrm{H}$-modes with Type I ELM edge, high $\beta_{\mathrm{p}} \mathrm{H}$-modes with Type II ELMs (pure and mixed with Type I), as well as from $\mathrm{H}$ mode plasmas with Type III ELM edge. The range of pedestal parameters where Type II ELMs appear is common with the other types of plasmas.

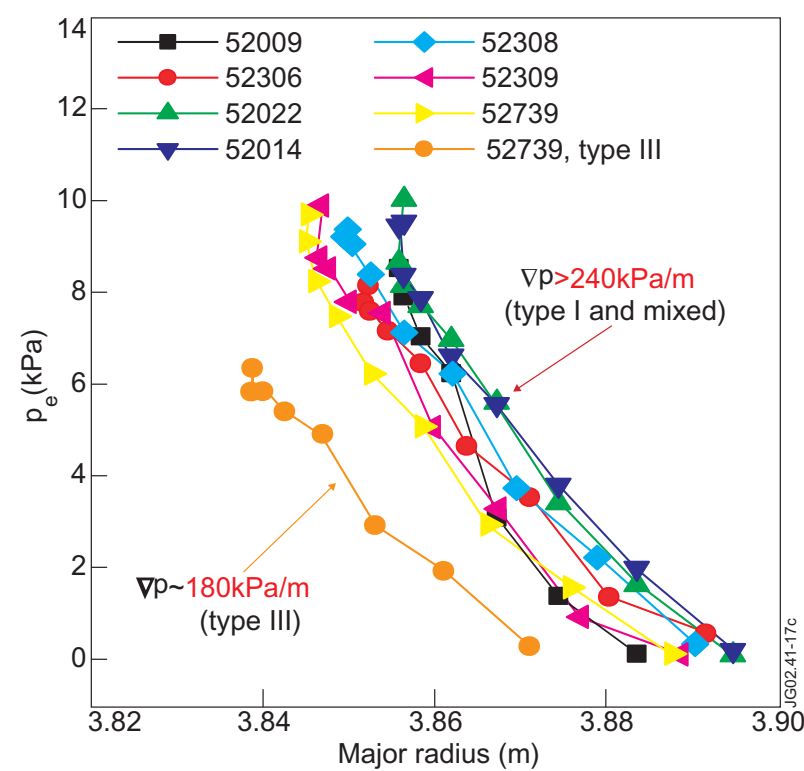

Figure 14. Pedestal electron pressure profiles calculated from the $n_{\mathrm{e}}$ and $T_{\mathrm{e}}$ profiles measured with the edge LIDAR system, for discharges in a density scan at $2.5 \mathrm{MA} / 2.7 \mathrm{~T}, \delta=0.47$. 
characteristics are all reminiscent of the signature of Type II ELMs in ASDEX Upgrade, and therefore, we propose the identification of the small, high frequency $\mathrm{D}_{\alpha}$ oscillations between Type I ELMs associated with the above phenomena as Type II ELMs.

One difference between the ASDEX Upgrade and JET operational conditions in Type II (or mixed) ELMs is the value of the edge safety factor. This difference is partly related to different operational constraints in the two machines: standard ELMy H-mode operations in ASDEX Upgrade are at $q_{95} \sim 4$, and the operational space at $q_{95} \sim 3$ is scarcely explored. Mixed Type I-II ELMy H-modes are normally observed in ASDEX Upgrade for $q_{95} \sim 4$, with pure Type II ELMs being obtained when $q_{95}$ is raised above 4.5 , all other conditions being equal. Recently, mixed Type I-II ELMs have been obtained in ASDEX Upgrade for $3.6 \lesssim q_{95} \lesssim 4$ in high power, high $\beta_{\mathrm{N}}$ and $\beta_{\mathrm{p}}$ plasmas (Sips et al 2002). For JET, the standard $q_{95}$ for ELMy H-mode operation is $\sim 3$, and operations at higher edge safety factor are restricted to low current plasmas, due to a lack of input power. Therefore, we explored the role of $q_{95}$ in obtaining Type II ELMs in two series of discharges at 2.0 MA/2.7 T and 1.5 MA/2.7 T, corresponding respectively to $q_{95} \sim 3.8$ and $\sim 5$. In both cases, the density scans were carried out with the same shape and $P_{\text {in }}$ as the higher current experiments. The one clear conclusion from the experiment at higher $q_{95}$ is that, in spite of the increase in the edge safety factor, no pure Type II ELMs were obtained. Nonetheless, mixed Type I and Type II ELMs were observed in the $I_{\mathrm{p}}=2.0 \mathrm{MA}$ scan, with the pedestal pressure having a very similar trend as in the higher $I_{\mathrm{p}}$ experiments, as shown in figure 15. One effect of increasing $q_{95}$ to 3.8 is that the transition to the mixed ELM regime occurs at lower normalized density and collisionality than for plasmas with $q_{95} \sim 3$ : in fact mixed Type I and II are observed at $n_{\text {ped }} \sim 5 \times 10^{19} \mathrm{~m}^{-3}$, that corresponds to approximately $60 \% n_{\mathrm{GR}}$ and to $v_{\text {neo }}^{*} \sim 0.6$ (compared to typical $v_{\text {neo }}^{*} \sim 0.7-0.8$ for the $q_{95} \sim 3$ cases). The MHD fluctuations observed between Type I ELMs at $q_{95} \sim 3.8$ are similar to those measured at the higher current, although the changes in the spectrum compared to the low density reference are not as pronounced as at high $I_{\mathrm{p}}$, leaving open the possibility that compound Type I-III ELMs (often observed at high density) maybe mixed with Type II ELMs. The experiment was not pursued further since the global confinement of these series of discharges was not as good as that of the reference series at $2.5 \mathrm{MA} / 2.7 \mathrm{~T}$, with $H_{98}$ decreasing from $\sim 1$ at low density to $H_{98} \sim 0.85$ at $n / n_{\mathrm{GR}} \sim 0.9$. The further increase in $q_{95}$ to $\sim 5$ lead to disappointing results, with the plasma undergoing a transition to Type III ELMs as soon as gas fuelling was applied, with the associated loss of confinement. One possible qualitative explanation of these results may come from the observation that Type II ELMs in JET are observed at pedestal $n_{\mathrm{e}}$ and $T_{\mathrm{e}}$ very near to the critical values for the Type I-III transition. It is possible that increasing the $q_{95}$ by reducing the plasma current, reduces the 'access window' for Type II ELMs (i.e. the onset of Type III ELMs moves towards higher $T_{\text {ped }}$ and lower $n_{\text {ped }}$ by increasing $q$ ) and Type III ELMs are destabilized first (Chankin and Saibene 1999). Nonetheless, these results are not fully understood, and more experiments are planned in the near future in JET to investigate further the relationship between Type II ELM access conditions and $q_{95}$.

3.1.1. Plasma energy losses with mixed Type I and Type II ELMs. Further insight on the characterization of Type II ELMs in JET is gained by comparing the global plasma power balance of plasmas with pure Type I ELMs and those with mixed Type I and Type II ELMs. This was carried out for the main density scan at $2.5 \mathrm{MA} / 2.7 \mathrm{~T}, \delta=0.47$. The terms considered in the power balance equation are: total input power $P_{\mathrm{TOT}}=P_{\text {in }}+P_{\Omega}$, bulk radiation, $P_{\text {rad }}^{\text {bulk }}$ and the average ELM energy losses associated with Type I ELMs, $P_{\mathrm{ELM}}^{\text {Type I }} . P_{\mathrm{ELM}}^{\text {Type I }}$ is calculated as the product of the time averaged Type I ELM prompt energy loss per ELM and the ELM 


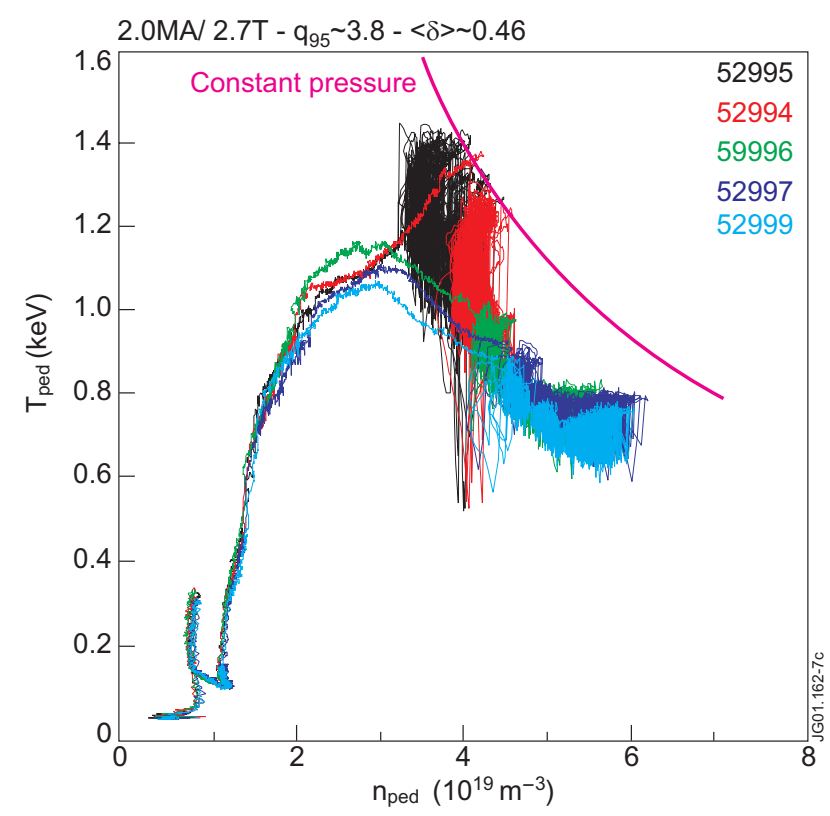

Figure 15. The $n_{\mathrm{e}}-T_{\mathrm{e}}$ diagram for the density scan at $2.0 \mathrm{MA} / 2.7 \mathrm{~T}\left(q_{95} \sim 3.8\right), \delta=0.46$. Mixed Type I-II ELMs are observed in pulses 52997 and 52999.

frequency, or $P_{\mathrm{ELM}}^{\text {Type I }}=\overline{\Delta W_{\mathrm{ELM}}} \times f_{\mathrm{ELM}}$, in MW. As pointed out above, the Type I ELM frequency goes down in the mixed Type I-II phases of these discharges and, at the same time, $\Delta W_{\mathrm{ELM}}$ is reduced compared to that of ELMs of the same $f_{\mathrm{ELM}}$ but occurring at lower $n_{\text {ped }}$ and higher $T_{\text {ped }}$. This 'decoupling' of the ELM energy losses and ELM frequency is described in detail in Loarte et al (2001b, 2002a), and is consistent with the ELM losses being determined by the plasma pedestal parameters, while the ELM frequency is mainly a consequence of the inter-ELM local confinement. Finally, the $\mathrm{d} W / \mathrm{d} t$ term (averaged over several $\tau_{\mathrm{E}}$ ) is neglected, since the power balance analysis is carried out at time slices in the discharge were the plasma stored energy is approximately constant.

As shown in figure 16, all the terms of the power balance are nearly constant across the scan, apart from $P_{\mathrm{ELM}}^{\text {Type I }}$ that decreases with the onset of mixed Type I and Type II ELMs. This observation clearly means that power losses between Type I ELMs have increased with the appearance of Type II ELMs, in the particular example considered, from $\sim 6$ to $\sim 10 \mathrm{MW}$. This result demonstrates, although indirectly, that Type II ELMs are associated with enhanced transport between ELMs. Unfortunately, no infrared camera measurement of the power density at the divertor plates during Type II ELMs in JET are available to date to confirm the power balance results, and supply more information on their energy deposition pattern (in time and space).

The persistence of Type I ELMs in the JET plasmas analysed above, although at reduced frequency, shows that the additional losses in-between ELMs are not sufficient to maintain the pedestal parameters in steady state. The comparison of the time evolution of $T_{\text {ped }}$ and $n_{\text {ped }}$ between Type I ELMs in absence and with Type II ELM activity, at constant input power, shows that the pedestal temperature comes near to saturation with Type II ELMs, while the density does not. In particular, the value of density rise rate $\mathrm{d} n_{\mathrm{ped}} / \mathrm{d} t$ is reduced by approximately a factor of two with Type II ELMs compared to a case of similar density with pure Type I ELM 


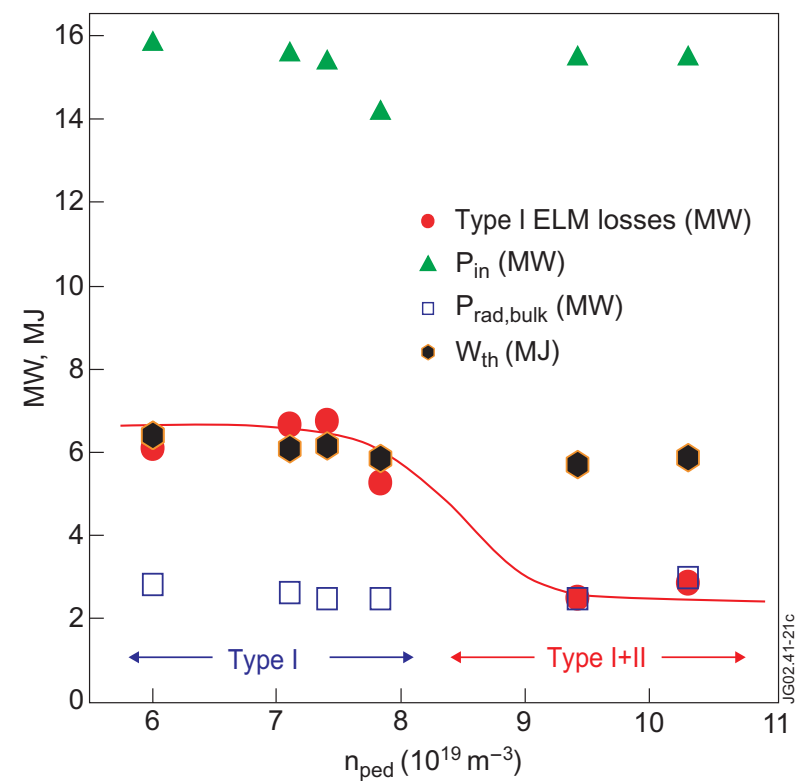

Figure 16. The radiation $P_{\text {rad }}^{\text {bulk }}(\mathrm{MW})$, ELM power losses $P_{\text {loss }}^{\text {Type I }}(\mathrm{MW})$, for the density scan at $\delta=0.47$ and 2.5 MA. The Type I power losses decrease at the onset of mixed Type I-II ELMs. The plasma stored energy is also shown for reference. Each point is representative of one discharge.

behaviour, although the total particle increase between ELMs, $\Delta n_{\text {ped }}$, between Type I ELMs remains approximately constant.

3.1.2. MHD and density fluctuations. As mentioned in section 3.1, Type II ELMs in JET are associated with a change in the MHD fluctuation spectrum (Becoulet et al 2001), observed only and always in association with the characteristic $\mathrm{D}_{\alpha}$ signature of Type II ELMs, so that MHD frequency analysis is used in JET to identify Type II ELMs. Figure 17 shows the MHD spectrograms for pulses 52008, with $n_{\text {ped }} \sim 60 \% n_{\mathrm{GR}}$ and Type I ELMs, together with that for pulse 52308, with $n_{\text {ped }} \sim 90 \% n_{\mathrm{GR}}$ and mixed Type I-II ELMs. The corresponding divertor $\mathrm{D}_{\alpha}$ signals are shown in figure 18, for the same time window of the MHD spectra.

The pattern of MHD fluctuations for pure Type I ELM discharges, such as 52008, and that associated with Type II ELMs as in 52308, can be clearly distinguished from the simple inspection of the spectrograms in figure 17. In particular, we see that Type II ELM phases are characterized by an increased intensity of broadband low frequency fluctuations, while at the same time the high frequency component is strongly suppressed. A more quantitative evaluation of the changes in the fluctuation spectra can be carried out by taking a number of Fourier spectra (i.e. a vertical cut in the spectrogram) in a time interval representative of the typical MHD of the pulse in-between Type I ELMs. Each spectrum is divided by the frequency of each sample, and then all the spectra in the selected time window are averaged. This analysis, shown in figure 19, provides quantitative information on the change in the MHD turbulence associated with Type II ELMs. In particular, from the comparison of the MHD in the period between Type I ELMs of pulses 52008 and 52308, we see that the MHD turbulence of 52308 is enhanced in the $10-30 \mathrm{kHz}$ frequency range by approximately a factor of four, while it is reduced by up to one order of magnitude for frequencies $\gtrsim 50 \mathrm{kHz}$. The main toroidal number of the low frequency MHD fluctuations is $n=-8$ (the negative sign means that the mode 

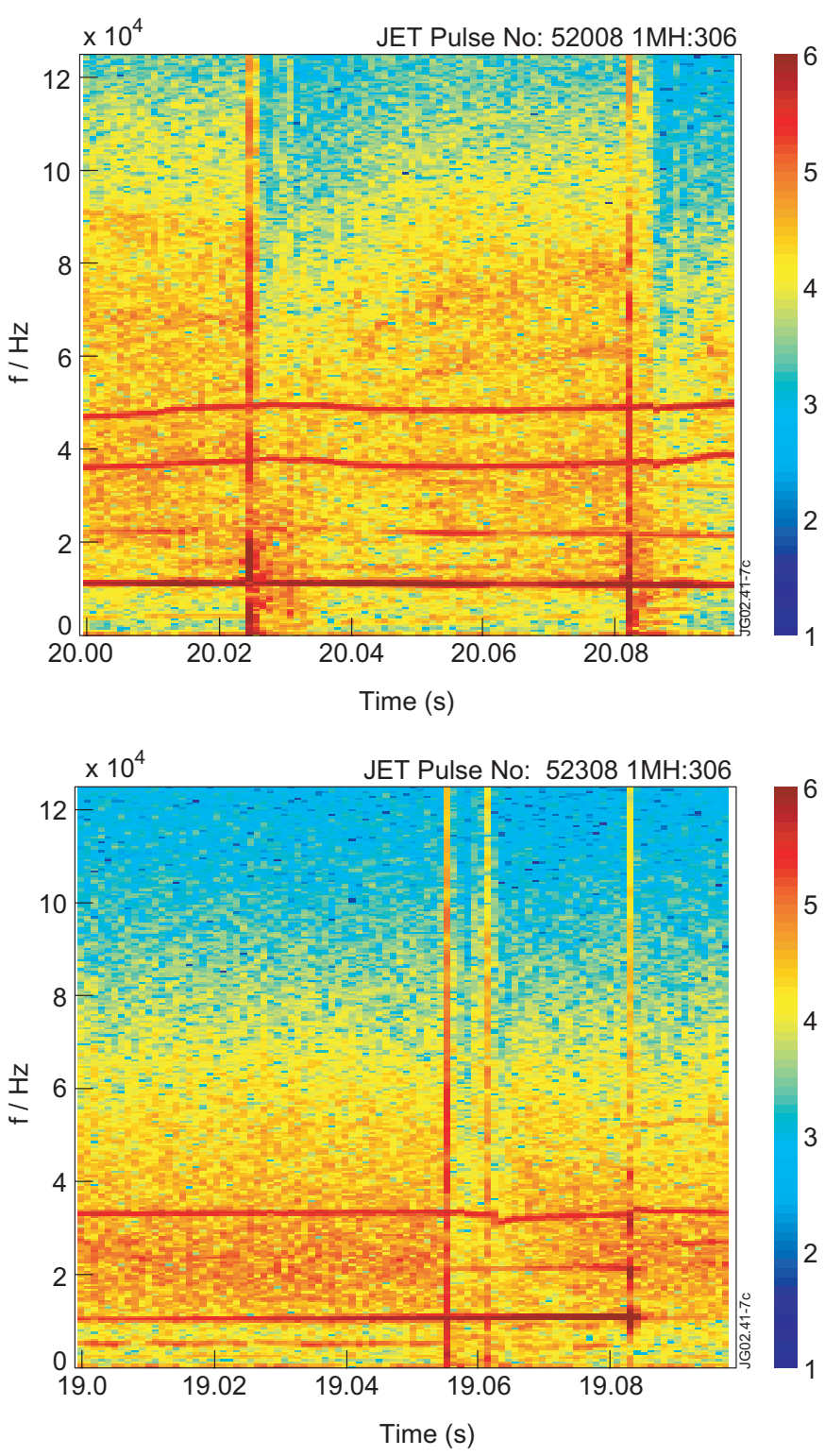

Figure 17. Frequency spectrum of Mirnov pick-up coil signal for pulses 52008 and 52308 $\left(2.5 \mathrm{MA} / 2.7 \mathrm{~T}, \delta=0.47, n_{\text {ped }} \sim 55 \% n_{\mathrm{GR}}\right.$ and $n_{\text {ped }} \sim 90 \% n_{\mathrm{GR}}$, respectively). The colour coding indicates the amplitude of the Fourier component normalized by the frequency on a logarithmic scale.

rotates in the $\mathrm{e}^{-}$diamagnetic direction) (Becoulet et al 2002).

Density fluctuation measurements for these pulses are also available by means of multiple fixed-frequency reflectometry. The range of frequencies of the reflectometer system correspond for these pulses to reflecting layers situated in the pedestal region, typically at positions $0.95 \lesssim \rho \lesssim 1$. The spectrograms of the density fluctuations $\tilde{n}_{\mathrm{e}}$ for the two discharges are shown in figure 20, for the same pulses and time windows of the MHD fluctuation measurements (refer to figure 18 for the $\mathrm{H}_{\alpha}$ emission traces for the two discharges) for a particular frequency, 

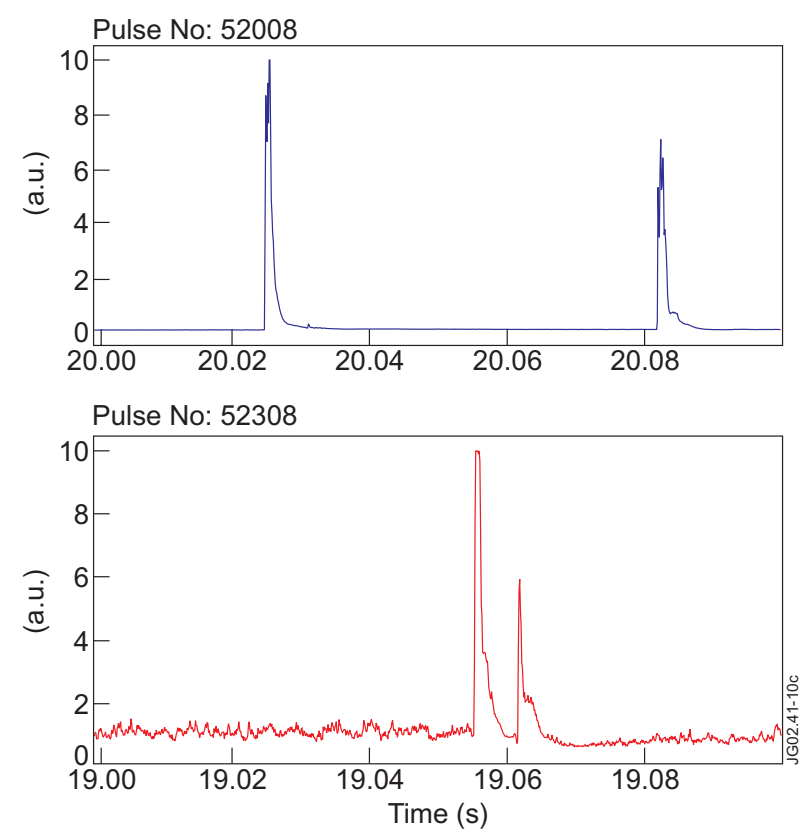

Figure 18. Divertor $\mathrm{D}_{\alpha}$ emission for pulses 52008 and 52308, on the same timescale as figure 17.

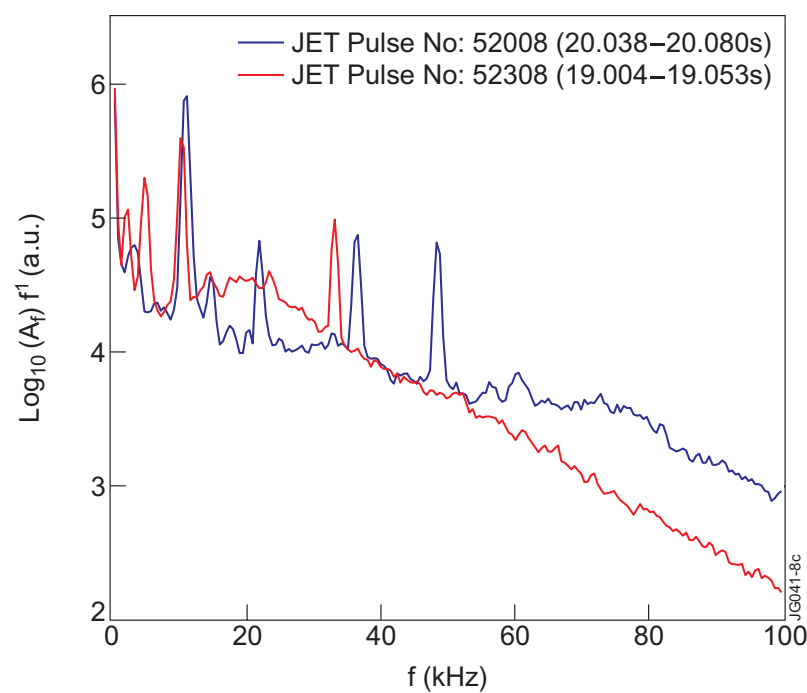

Figure 19. Comparison of the intensity of MHD turbulence for pulses 52008 and 52308. The sharp peaks come to form a continuous narrow band MHD, for both pulses (see also figure 17). The $y$-axis is in arbitrary units.

corresponding to a density $\sim 4 \times 10^{19} \mathrm{~m}^{-3}$. As for the case of the MHD turbulence, we see that Type II ELM activity is associated with an increase of the $\tilde{n}_{\mathrm{e}}$ on a similar frequency range as the MHD fluctuations. The density fluctuations spectra shown in figure 20 have been analysed and compared by calculating the raw complex amplitude of the reflectometer spectra $\left(\mathrm{e}^{i \phi}\right)$, over the same time window used for the analysis of the MHD fluctuations in figure 19 . The 
results of this analysis, summarized in figure 21, shows that Type II ELMs are associated with an increase of the density fluctuations in the frequency range $10-40 \mathrm{kHz}$.

Both MHD turbulence and the $\tilde{n}_{\mathrm{e}}$ measurements indicate that Type II ELMs could be associated with a change in the energy and/or particle transport across the ETB. Turbulent transport could possibly be providing the loss channel required to explain the changes in the global power balance of JET ELMy H-modes with mixed Type I and Type II ELMs, described in section 3.1.1. However, measurements of the turbulent particle flux are required to substantiate this hypothesis.

The results of the fluctuation analysis of Type II ELMs in JET is qualitatively similar to that reported for Type II ELMs in ASDEX Upgrade (Stober et al 2001a). In both machines, the MHD turbulence analysis shows an increase of the broadband turbulence around the $30 \mathrm{kHz}$ frequency, with estimated toroidal $n$ number $\sim 4$ for ASDEX Upgrade, as well as increased density fluctuations in the pedestal region. On the other hand, recent MHD stability calculations for the Type II ELMy discharges in ASDEX Upgrade (Horton 2002) finds that the toroidal mode number of the most unstable mode is $n \gtrsim 8$. Further analysis of both ASDEX Upgrade and JET data is needed to carry out a detailed comparison of the MHD behaviour in the two experiments.

The characteristic pattern of the turbulence observed in JET with Type II ELMs is quite different from that of the C-Mod EDA mode (Hubbard et al 2001, Snipes et al 2001). In C-Mod, both MHD and density fluctuation are enhanced during the EDA phase and are claimed to provide the required transport across the pedestal to maintain steady state plasma parameters with a ETB and in absence of ELMs. On the other hand, the mode observed in C-Mod occurs in a much narrower frequency band than both in JET and in ASDEX Upgrade, showing a more coherent character (it is in fact called a quasi-coherent mode, or QC mode). Recent

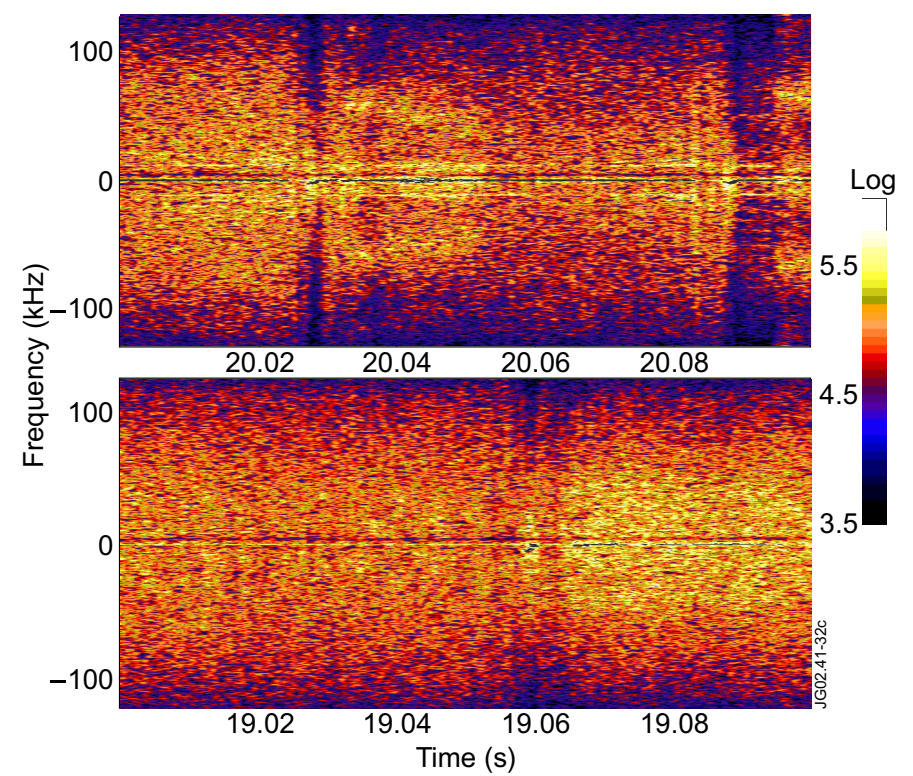

Figure 20. Spectrogram of density fluctuations calculated applying a moving average FFT to the signals from reflectometry, for pulses 52008 (above) and 52308 (below). The time window for pulse 52008 includes two Type I ELMs. For discharge 52308, the spectrum includes two phases with Type II ELM, separated by a Type I ELM at $\sim 19.55 \mathrm{~s}$. The corresponding divertor $\mathrm{D}_{\alpha}$ emissions are shown in figure 18 . 


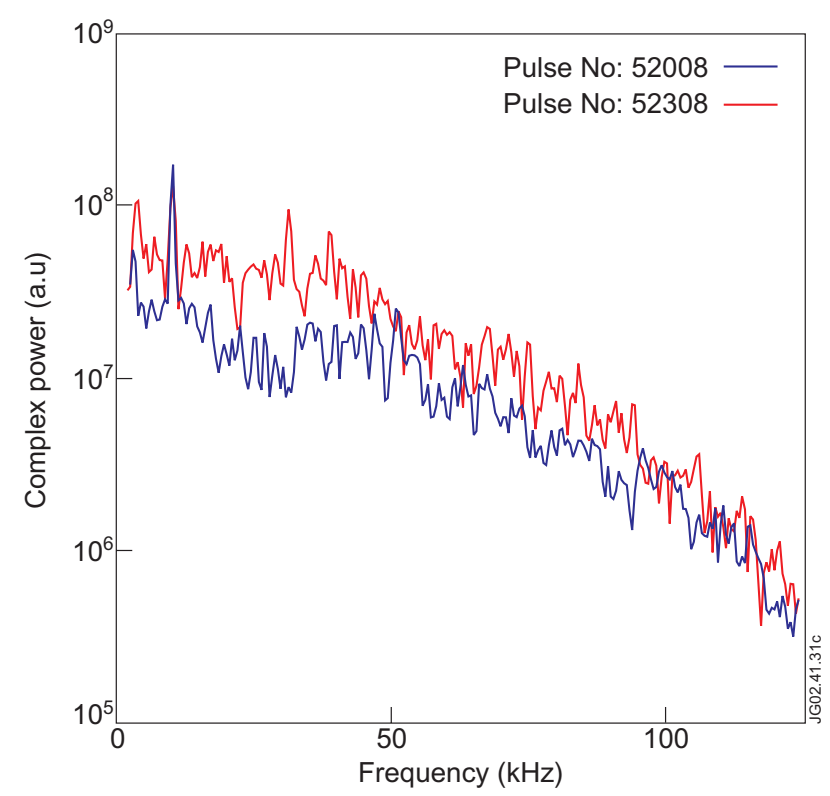

Figure 21. Comparison of the intensity of density fluctuations in pulses 52008 and 52308 ( $y$-axis in arbitrary units).

results (Mossessian et al 2002), show that a transition from EDA to H-modes with small, high frequency ELMs is obtained in C-Mod by increasing the input power. In this case, the QC mode disappears and is replaced by broadband MHD, in the frequency range $\lesssim 30-40 \mathrm{kHz}$, similar to the observations in ASDEX Upgrade and JET.

\subsection{Effects of the lower triangularity}

The effect of plasma shape on the pedestal and ELM properties, as well as on global confinement at high density, was also investigated by comparing plasmas with the same upper $\delta$, but with different lower $\delta$. The first density scan used in the comparison is the 2.5 MA/2.7 T, $\delta=0.47$, already described in detail in this paper. The second one was carried out at the same $I_{\mathrm{p}}$ and $B_{\mathrm{t}}$ and similar NB input power (15 versus $16.5 \mathrm{MW}$ ) The EFIT reconstruction of the two configurations is shown in figure 1. Although the geometry of the two configurations is quite different near the $\mathrm{x}$-point ( $\delta_{\text {low }}$ is reduced from $\sim 0.4$ to $\sim 0.3$ by moving the $\mathrm{x}$-point to the low field side by $\sim 10 \mathrm{~cm}$ ), the variation in the average equilibrium properties at the plasma edge is small, with the shear and $q$ calculated at $95 \%$ of the flux differing by $<10 \%$. Nonetheless, the plasmas with higher $\delta_{\text {low }}$ (and higher average $\delta$ ) achieve higher confinement at higher density, as well as higher pedestal pressures.

A comparison of the $n_{\mathrm{e}}-T_{\mathrm{e}}$ diagram for the two gas/density scans is found in figure 11, were the data for the lower $\delta_{\text {low }}$ density scan are represented by the red symbols (squares for Type I ELMs, a star for the Type III point). We see that the lower $\delta_{\text {low }}$ plasmas have, for a given $n_{\text {ped }}$, lower $T_{\text {ped }}$, and achieve a smaller $p_{\text {ped }}$ across the density range. Moreover, the maximum $n_{\text {ped }}$ achieved with Type I ELMs by the plasmas with high $\delta_{\text {low }}$ is $\sim 10 \%$ higher than that of the low $\delta_{\text {low }}$ plasmas. 


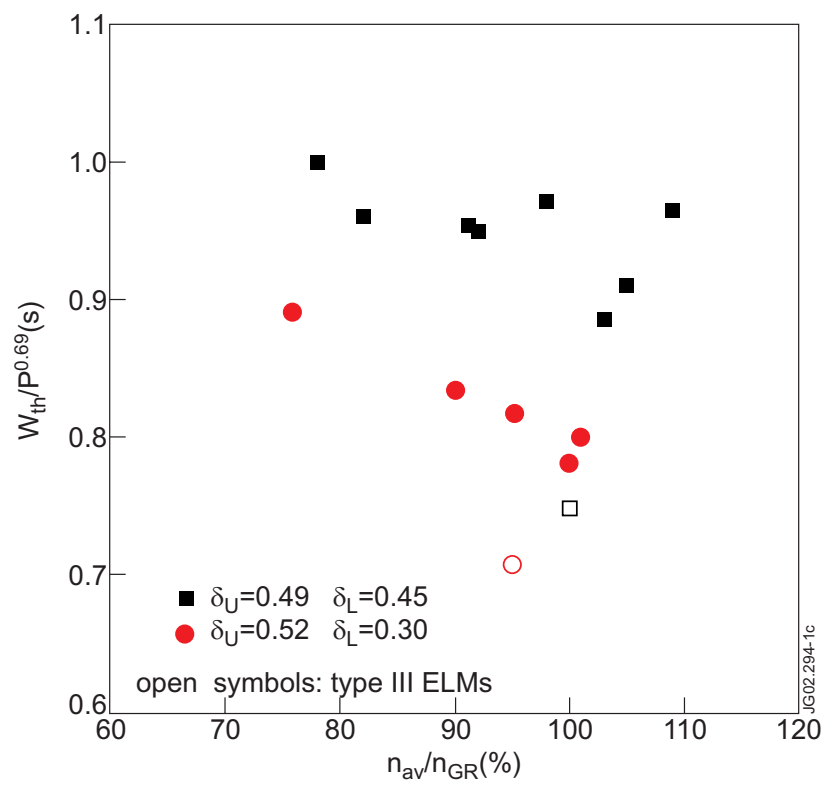

Figure 22. Comparison of $W_{\text {th }}$ for the two density scans at high and low $\delta_{\text {low }}$ (black squares and red circles, respectively), as function of the line average density normalized to the Greenwald density. $W_{\text {th }}$ is normalized to $P_{\text {in }}^{-0.69}$ to account for the difference in input power (the exponent of $P_{\text {in }}$ is the power degradation of the $H_{98}$ scaling).

Mixed Type I-II ELMs are observed also for the low $\delta_{\text {low }}$ plasmas, at pedestal density $\sim 80 \% n_{\mathrm{GR}}$, although the change in ELM behaviour occurs even nearer (in terms of pedestal parameters) to the Type I-III transition, and is sustained in a reduced $n_{\text {ped }}$ window. Moreover, moving the $\mathrm{x}$-point to the low-field side of the machine seems to have a strong effect on both the Type I ELM frequency and on the prompt ELM energy losses $\Delta W_{\mathrm{ELM}}^{\mathrm{N}}=\Delta W_{\mathrm{ELM}} / W_{\text {ped }}$. In particular, at high pedestal density $\left(n_{\text {ped }} \sim 90 \% n_{\mathrm{GR}}\right.$, or $\left.\sim 0.9 \times 10^{20} \mathrm{~m}^{-3}\right), f_{\mathrm{ELM}} \sim 40 \mathrm{~Hz}$ for $\delta_{\text {low }} \sim 0.3$, to be compared to $f_{\mathrm{ELM}} \sim 12 \mathrm{~Hz}$ for $\delta_{\text {low }} \sim 0.45$. The ELM energy losses are also quite different in the two cases, with $\Delta W_{\mathrm{ELM}}^{\mathrm{N}} \sim 4-5 \%$ for the low $\delta_{\text {low }}$ plasmas, while in the case of the high $\delta_{\text {low }}$ configurations, the minimum $\Delta W_{\mathrm{ELM}}^{\mathrm{N}}$ is still of the order of $8-9 \%$. As described in detail in Loarte et al (2001a, 2002a), the drop in pedestal temperature associated with a Type I ELM decreases with density, while the ELM particle losses are approximately constant for increasing $n_{\text {ped }}$. In particular, the ELMs with $\Delta W_{\mathrm{ELM}}^{\mathrm{N}} \sim 4-5 \%$ mentioned above occur without any associated drop in $T_{\text {ped }}$, and therefore are 'pure particle' ELMs. Recent analysis of a multi-machine database of Type I ELM energy losses (Federici et al 2002, Loarte et al 2002b) shows that $\Delta W_{\mathrm{ELM}}^{\mathrm{N}}$ between $5 \%$ and $10 \%$ could be acceptable for the ITER Tungsten divertor. ELM energy losses of $\sim 5 \%$, well within the acceptable values for ITER, are obtained in the low $\delta_{\text {low }}$ plasmas. These small 'pure particle' ELMs are obtained at a somewhat reduced global confinement compared to the values obtained in the high $\delta$ discharges, whose Type I ELMs of 8-9\% are only marginally within the maximum allowable $\Delta W_{\mathrm{ELM}}^{\mathrm{N}}$ projected to ITER. The thermal plasma stored energy content for the two density scans is compared in figure 22 , and the reduction of $W_{\text {th }}$ for the low $\delta_{\text {low }}$ and its trend with density are consistent with the reduced $p_{\text {ped }}$.

The analysis of experiments in DIII-D (Lao et al 1999, Ferron et al 2000), highlights the importance of the plasma geometry on edge stability, in particular on access to second ballooning mode stability, and may provide some indication for the interpretation of the effect 
of the lower $\delta$ (or of the x-point radial position) on the ELMs and $p_{\text {ped }}$ in JET. The DIII-D analysis indicates that variation on local strength of the poloidal field in the bad curvature region of the plasma can open/close access to second stability for ballooning modes, modify the edge bootstrap current build-up and therefore change the dominant MHD, affecting the maximum pedestal pressure, ELM size and frequency.

\subsection{Discussion}

As mentioned in the introduction, plasmas with good global energy confinement and small ELMs have been observed in Alcator C-Mod, DIII-D, JT-60U, ASDEX Upgrade and JET, although in JET pure Type II ELMs plasmas have not yet been obtained. A brief comparison of the access conditions and characterization of small ELM regimes in different machines may help to clarify the mechanisms leading to the suppression of Type I ELMs and the onset of the Type II. The case of the 'grassy' ELMy H-modes in C-Mod is somewhat different from the others, since Type I ELMy H-modes are not observed in that machine, and it is not discussed in detail.

The first observation of a reduction in the Type IELM amplitude at high plasma shaping and high density was reported by Ozeki et al (1990) in DIII-D. The change in the ELM behaviour was attributed to the plasma pedestal accessing the connection regime between first and second stability to $\infty-n$ ballooning modes. Very high plasma shaping $(\delta \gtrsim 0.4$ and $\kappa \gtrsim 1.8)$ as well as very high safety factor $\left(q_{95} \sim 7\right)$ were required to approach the second stable regime. Unfortunately, no details on the pedestal parameters are reported in that publication, nor is an assessment of any change in the ELM energy losses (compared to standard Type I ELMs) available.

In the case of JT-60U (Kamada and the JT-60U Team 2001, Kamada et al 2002), $\delta \gtrsim 0.6$, $\beta_{\text {pol }} \gtrsim 1.6$ and $q_{95} \gtrsim 4$ are required to obtain 'grassy' or Type II ELMy edge in steady state. Detailed mapping of the $\delta-q_{95}$ operational space in JT-60U has shown that, at fixed $\beta_{\mathrm{pol}}$, a reduction of the plasma triangularity results in an increase of the minimum $q_{95}$ required to access the small ELMs regime. As shown in figure 13, the pedestal parameters of Type II ELM discharges in JT-60U are virtually the same as standard Type I ELMy H-modes, typically low density $\left(n_{\text {ped }} \sim 30 \% n_{\mathrm{GR}}\right)$ and high temperature, corresponding to a low collisionality regime $\left(v_{\text {neo }}^{*} \sim 0.1\right)$.

The access to the Type II ELMy regime in ASDEX Upgrade (Stober et al 2001a) requires a quasi-double-null plasma configuration, high $\delta$ (typically $\gtrsim 0.4)$, high density $\left(n_{\mathrm{e}} \gtrsim 85 \% n_{\mathrm{GR}}\right.$, $v_{\text {neo }}^{*} \sim 2$ ) and $q_{95} \gtrsim 4-4.5$, with mixed Type I and II ELMs observed for $q_{95}$ values as low as 3.5 (Sips et al 2002). Both JT-60U and ASDEX Upgrade have demonstrated that the suppression of Type I ELMs is gradual as the critical parameters are approached, with phases where Type I and Type II can coexist. In both machines, the transition from Type I to Type II occurs at approximately constant $p_{\text {ped }}$ and $\nabla p_{\text {ped }}$.

For the case of JET, we have shown that mixed Type I-II ELMy H-modes are obtained at high $\delta$ and high pedestal density, in a rather narrow edge operational corner, near to the Type I-III ELM boundary. As discussed in Sartori et al (2002), access to the mixed ELM regime has also been observed in JET for a reduced $\delta \sim 0.33, q_{95} \sim 3.3$ and high input power.

From the above observations, high shaping and edge shear seems to be the one common feature for access to Type II ELMs for all experiments; this is achieved by a combination of triangularity and elongation, proximity to double null configuration or/and distortion of the flux surfaces by Shafranov shift at high $\beta_{\text {pol }}$. The central role of magnetic shear and edge safety factor points to a change in the MHD stability in the pedestal region being associated with the onset of Type II ELMs. Modelling of JT-60U Type II ELMy plasmas (Lao et al 2001) as well 
as recent numerical studies (see for instance Wilson et al (2001)) show that $p_{\text {ped }}$, changes in ELM frequency and the size of the ELM losses can be related to access to second stability to $\infty-n$ ballooning modes and to changes in the leading unstable MHD mode in the pedestal region. This picture is consistent with the results of $\infty-n$ ballooning analysis by Korotkov et al (2000), showing that pedestal pressure gradients in excess of the first stability limit are obtained in some high $\delta$ JET plasmas.

Some results of recent MHD stability analysis of JET discharges (Huysmans 2001) at high $\delta$ and density with mixed Type I-II ELMs are illustrated in figure 23, summarizing the results of the MHD analysis carried out with the MISHKA code using inputs from JETTO simulations of two JET ELMy H-modes, at low and high $\delta(50460, \delta=0.23$ on the top and $52308, \delta=0.47$ on the bottom). In the low $\delta$ case, the stable operating space (in current density/pressure gradient) is reduced compared to the high $\delta$ discharge, with reduced stability to ideal $\infty-n$ ballooning modes as well as to intermediate $n$ kink modes.

At high $\delta$, the operating space opens up, for both ballooning as well as kink modes, but the access to second stability is essentially precluded by low $n$ kink modes. This result for the high $\delta$ plasmas is critically dependent on the estimation of the edge bootstrap current profile $J_{\mathrm{bs}}(r)$. This is calculated from the experimental $n_{\mathrm{e}}$ (and $T$ ) pedestal profiles, and then varied in the calculations to test the sensitivity of the MISHKA code results. The effect on stability of decreasing the edge bootstrap current is schematically represented by the arrow in the lower box of figure 23, with the low $n$ kink mode stability boundary moving to the right of the diagram. Therefore, at lower $J_{\mathrm{bs}}$, the plasma pedestal is stable to low $n$ kink modes, and the most unstable modes are intermediate $n$ ballooning/peeling modes. These modes close the access to the second stable regime to $\infty-n$ ballooning modes, although the maximum stable $\nabla p$ is above the first ballooning stability limit. These calculations suggest that the pressure limit across the pedestal may increase by a factor of two, albeit for a narrow range of $J_{\text {bs }}(r)$. This result of the simulations is consistent with the variation in pedestal height observed in JET experiments at high plasma $\delta$. Finally, note that the MISHKA calculations show that the combination of high density and a broad density profile may even stabilize the kink modes completely.

The measurement of the edge density profile is usually not very accurate in JET, in particular for high $\delta$ plasmas (see section 1.2), although good Li beam measurements are available for a small number of the discharges studied in this paper. An example of such measurements is shown in figure 24 , which compares density profiles for a pure Type I ELM discharge (pulse 53298) with those of a pulse with mixed Type I and Type II ELMs (53299). These measurements show clearly the dramatic variations of the edge density gradients with density in high $\delta$ discharges. The increase in $n_{\text {ped }}$ not only corresponds to a steepening of the profiles, but the region of the steepest gradient moves outside, towards the separatrix. Such changes in gradient and shape of the edge density modify the $J_{\mathrm{bs}}(r)$, which, as shown above, has a strong influence on stability. More accurate and systematic measurement of the pedestal profiles are required in order to make a detailed comparison with theoretical and numerical predictions. In the meantime, one can speculate that the changes in the density profiles at high gas fuelling, possibly due to the reduction of the ionization mean free path, influence $J_{\mathrm{bs}}(r)$ in such a way that the pedestal accesses an 'optimum' stability domain, where intermediate-high $n$ modes may provide a trigger mechanism to expel heat and particles in small bursts. For the case of mixed Type I-II ELMs, the effect of these modes is to reduce the rate at which the pedestal pressure builds up, and delay the access to the stability domain where Type I ELMs become unstable. It is also possible, of course, that turbulent transport itself is providing the mechanism by which Type I ELMs are delayed or suppressed. 

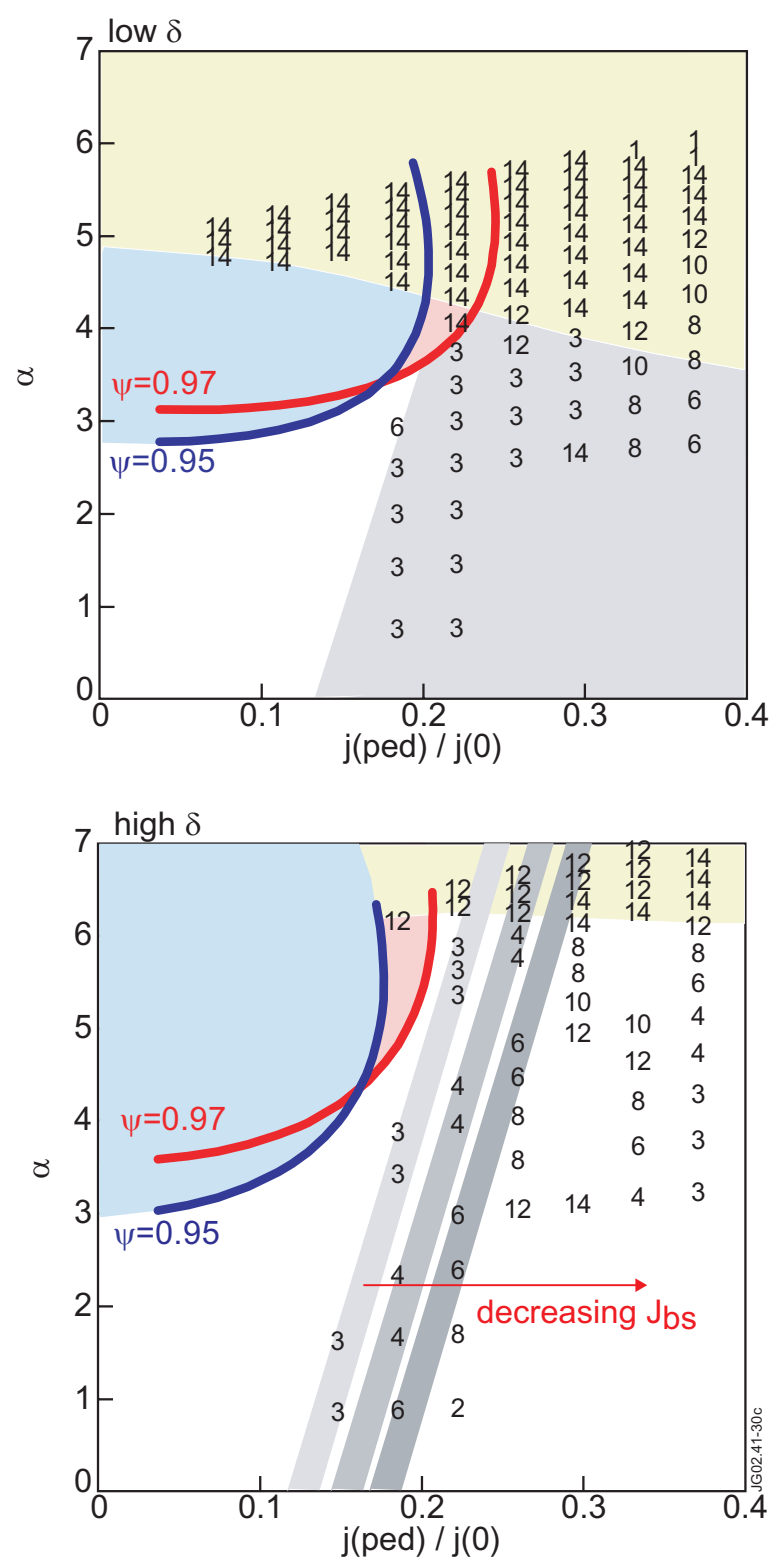

Figure 23. Comparison of the MHD stability space for two JET ELMy H-modes, at low $\delta$ (upper box, pulse 50460, $\delta=0.23$ ) and high $\delta$ (lower box, pulse 52308, $\delta=0.47$ ). The input parameters for the MISHKA calculations are provided by JETTO interpretative simulations of the two discharges. At low $\delta$, the maximum pedestal pressure gradient is limited by $\infty-n$ ballooning and low $n$ kink modes. Increasing $\delta$ opens up the operational space and the stability limit is given by low-intermediate $n$ ballooning modes. The effect of reduced bootstrap current in the pedestal region on stability, schematically shown by the red arrow in the lower figure, is to widen the access to the connection region between first and second $\infty-n$ ballooning stable region. The $\infty-n$ unstable region is indicated by the blue and pink colour (depending on the field line), the high $\mathrm{n}$ ballooning are in the zone in yellow, while kink-peeling modes are indicated with shades of grey. 

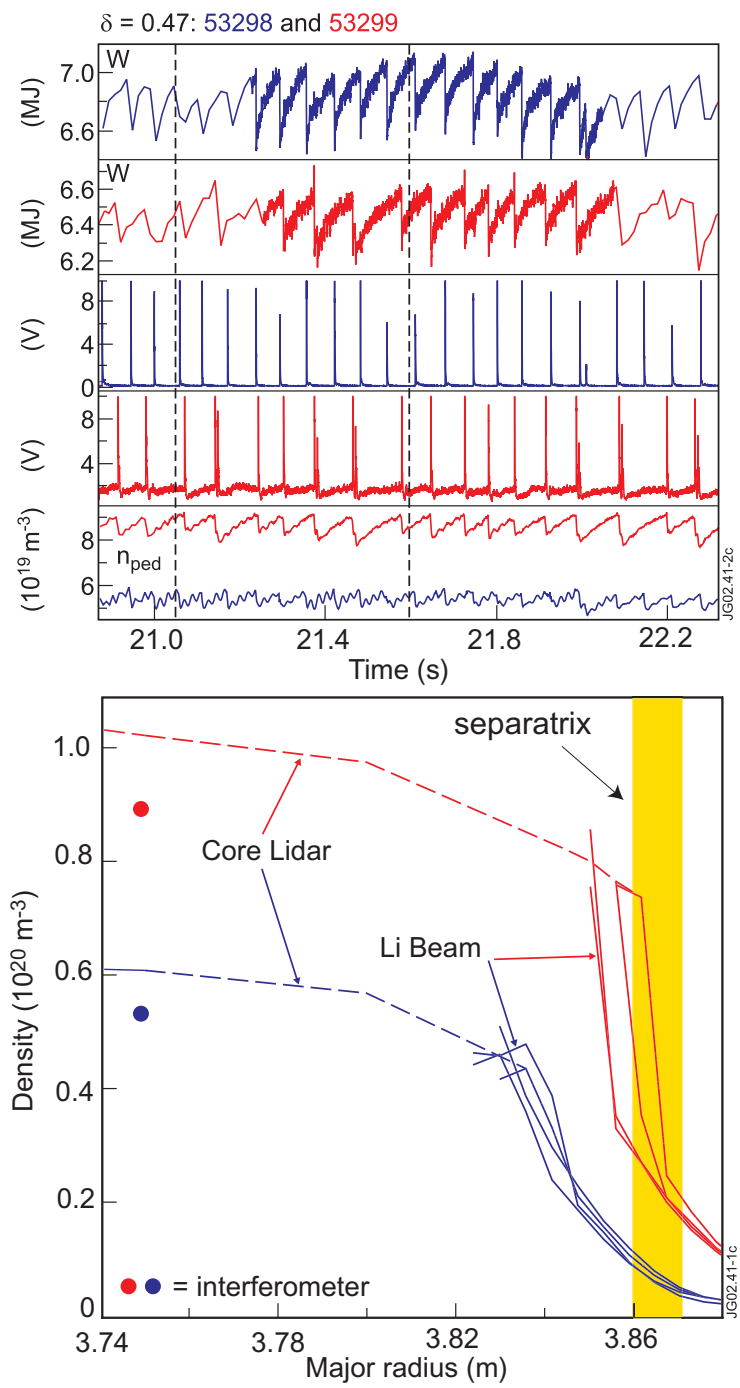

Figure 24. Li beam density profiles for pulses 53298 (blue) and 53299 (red), 2.5 MA/2.7 T, $\delta=0.47, P_{\text {in }}=15 \mathrm{MW}$. The dotted lines in the top figure mark the time window in which the profiles have been taken. The time traces show the total plasma stored energy $W(\mathrm{MJ})$, the divertor $\mathrm{D}_{\alpha}$ emission and the pedestal density $n_{\text {ped }}\left(10^{19} \mathrm{~m}^{-3}\right)$. The Li beam density profiles (lower traces) are plotted with the core edge LIDAR profiles and the interferometer density measured at the outermost channel for comparison. The position of the separatrix is indicated by the band at $3.86 \mathrm{~m}$.

\section{Conclusions}

The positive effect of plasma shaping for achieving high density with high confinement in ELMy H-modes has been confirmed and extended in recent JET experiments. Plasmas with $\delta=0.47$, close to the ITER specifications, have achieved the core performances required for the $Q=10$ operation in ITER, in terms of the normalized parameters $n / n_{\mathrm{GR}}, H$ and $\beta_{\mathrm{N}}$. More specifically, the high $\delta$ plasmas have reached simultaneously $n \sim 90 \% n_{\mathrm{GR}}, H_{\mathrm{H}}=1$ and $\beta_{\mathrm{N}} \sim 2$, with high plasma purity $\left(Z_{\text {eff }}=1.4\right)$ and at low safety factor $q_{95}=3$. The absolute 
plasma temperature of these high density discharges is of course quite low, in JET and all other existing experiments. Extension of these high density regimes in JET to higher plasma current and input power would provide further confirmation of the robustness of these results.

The plasma density profiles at high density show a modest peaking, typically $\sim 1.2$ or less; moreover, the density profile peaking is found to be independent of plasma triangularity, indicating that the increase in the plasma energy content with $\delta$ is not due to density peaking. These observations give confidence on the potential for extrapolation to ITER of the positive effects of plasma shape observed in JET and other Tokamaks.

The maximum pedestal density achievable in JET also increase with plasma triangularity and, for the first time in JET, plasmas with $n_{\text {ped }} \sim n_{\mathrm{GR}}$ have been obtained, at constant plasma stored energy and high pedestal pressure. At $n_{\text {ped }} \sim 70 \% n_{\mathrm{GR}}$ and constant power flux across the separatrix, the Type I ELM frequency stops increasing with density and, if $n_{\text {ped }}$ is increased further, it even decreases. At the same time, small high frequency fluctuations are observed in the $\mathrm{D}_{\alpha}$ emission between ELMs. This change in the $\mathrm{D}_{\alpha}$ emission is accompanied by a characteristic and specific broadband MHD turbulence and by an increase of the density fluctuations in the pedestal region. A power balance analysis shows that the appearance of this inter-ELM activity is coincident with an increase of the plasma power losses between ELMs, while the pedestal pressure remains high. These observations, and the comparison with recent results from ASDEX Upgrade, suggest that the high frequency oscillations on the divertor $\mathrm{D}_{\alpha}$ emission could be identified as Type II ELMs. So far in JET, complete Type I ELM suppression at high pedestal pressure has not yet been achieved. The mixed ELM regime has been observed for $n_{\text {ped }} \gtrsim 70-80 \% n_{\mathrm{GR}}$, with $T_{\text {ped }}$ marginally above the critical temperature for the transition to Type III ELMs and $p_{\text {ped }}$ similar or higher to comparable pure Type I ELMy H-modes at similar density.

Finally, we have shown that changes in the plasma lower triangularity affect the ELM losses and the pedestal parameters, and in turn, the global confinement. In particular, plasmas with reduced $\delta_{\text {low }}$ have very low ELM energy losses (normalized to $W_{\text {ped }}$ ) at high density, of amplitude well within the acceptable losses projected to the Tungsten divertor of ITER.

The relevance of Type II ELMy H-modes for ITER operation is clear, but more work is required to identify the key parameters for access and to extrapolate this ELM regime to ITER. Moreover, more experimental information and modelling are required to extrapolate the magnitude of the energy and particle losses associated with Type II ELMs to ITER conditions (in particular to low pedestal $v^{*}$ ), and evaluate if Type II ELMs can provide an adequate exhaust mechanism for ITER H-mode plasmas. Experiments are planned in the 2002 JET experimental campaign to explore further the Type II ELM regime, in particular to achieve and document high performing H-modes with a pure Type II ELMs edge with both NB and ICRF heating, as well as to explore further the role of the plasma boundary geometry.

\section{Acknowledgments}

The authors thank all the JET-EFDA collaborators for the help in the execution of the experimental programme and in the data analysis. One author (G Saibene) thanks A Hubbard and D Mossessian (MIT), Y Kamada (JAERI), A A C Sips (IPP) and G Janeschitz (ITER) for the kind help and very interesting discussions. Figure 13 was produced with JT-60U data kindly provided by Y Kamada. The experiments described in this paper were carried out in the JET-EFDA Task Force S1, and the efforts of the Task Force leader J Ongena in the organization of the experiments, data analysis and publication of results are gratefully acknowledged. 


\section{References}

Aymar R et al 2001 ITER-FEAT: the future international plasma burning experiment-overview Proc. 8th Fusion Energy Conf. 2000 (October 2000) paper OV/1 (Vienna: IAEA) CD ROM ISSN 1562-4153

Becoulet M et al 2001 Edge fast magnetics measurements during ELMs of different types in JET Presented at IAEA TCM on Divertor Concepts (Aix en Provence, France, 2001)

Becoulet M et al 2002 Plasma Phys. Control. Fusion 44 A103

Beurskens M N A et al 2001 Analysis of plasma edge profiles at JET Proc 28th Conf. on Control. Fusion Plasma Phys. (Madeira) Europhys. Conf. Abstracts 25A 1229

Brix M et al 2001 Determination of edge density profiles in JET using a $50 \mathrm{keV}$ lithium beam Proc. 28th Conf. on Control. Fusion Plasma Phys. (Madeira) Europhys. Conf. Abstracts 25A 389

Chankin A V and Saibene G 1999 Plasma Phys. Control. Fusion 41913

Connor J W 1998 Plasma Phys. Control. Fusion 40191

Conway G D, Vayakis G, Fessey J A and Bartlett D V 1999 Rev. Sci. Instrum. 703921

Cox M 1988 FOKMEG: a package to determine the fast ion distribution function for the JET interpretation code suite Technical Report (Report on a JET Contract) KR5-33-04 UKAEA Culham Laboratory, UK

DIII-D Team 1990 Plasma Phys. Control. Nucl. Fusion Res. 169 IAEA-CN-53/A-I-4

Federici G et al 2001 J. Nucl. Mater. 290-293 260

Federici G et al 2002 Key ITER plasma edge and plasma-material interaction issues 15th PSI Conf. (Gifu, Japan, May 2002) J. Nucl. Mater. at press

Ferron J R et al 2000 Nucl. Fusion 71411

Horton L D 2002 Pedestal physics at ASEDX Upgrade Proc. 29th Conf. on Control. Fusion Plasma Phys. (Montreux, Switzerland) paper P2.047

Hubbard A E et al 2001 Phys. Plasmas 82033

Hutchinson I et al 2001 Nucl. Fusion 411391

Huysmans G T A 2001 Overview of MHD stability in edge transport barriers Presented at the 9th European Fusion Physics Workshop (Saariselka, Finland)

ITER Physics Basis 1999 Nucl. Fusion 392175 (by the ITER physics expert groups on confinement and transport and confinement and modelling and database)

Kamada Y et al 1996 Fusion Energy 1247 IAEA-CN-64/A1-6

Kamada Y et al 2002 Plasma Phys. Control. Fusion 44 A279

Kamada Y and the JT-60U Team 2001 Nucl. Fusion 411311

Korotkov A A et al 2000 Edge plasma pressure profile evolution in Type I ELM discharges on JET Proc. 2000 Int. Congress on Plasma Physics and 27th European Physical Society Conf. on Controlled Fusion and Plasma Physics Europhys. Conf. Abstracts 24B 1581

Lao L L et al 1999 Nucl. Fusion 11 Y1785

Lao L L et al 2001 Nucl. Fusion 41295

Leonard A W et al 2001 J. Nucl. Mater. 290-293 1097

Loarte A et al 2000 Comparison of high recycling H-modes on Alcator C-Mod and JET Bulletin of the 42nd Annual Meeting of the American Physics Soc. (Div. Plasma Physics) paper DO1/4

Loarte A et al 2001a Type I energy and particle losses in JET ELMy H-Modes Proc 28th Conf. on Controlled Fusion and Plasma Physics (Madeira) Europhys. Conf. Abstracts 25A 945

Loarte A et al 2001b Predicted ELM energy losses and power loading in ITER-FEAT Proc. 18th Fusion Energy Conf. 2000 (October 2000) paper ITERP/11 (Vienna: IAEA) CD ROM ISSN 1562-4153

Loarte A et al 2002a Characteristics and scaling of energy and particle losses during Type I ELMs in JET H-modes Plasma Phys. Control. Fusion 441815

Loarte A et al 2002b ELM energy and particle losses in present tokamaks and their extrapolation to burning plasmas experiments 15th PSI Conf. (Gifu, Japan, May 2002) J. Nucl. Mater. at press

Meade D M et al 2001 Mission and design of the fusion ignition research experiments (FIRE) Proc. 18th Fusion Energy Conf. 2000 (October 2000) paper FTP2/16 (Vienna: IAEA) CD ROM ISSN 1562-4153

Mossessian D A et al 2002 Plasma Phys. Control. Fusion 44423

Nordman H, Strand P, Weiland J and Christiansen J P 1999 Nucl. Fusion 391157

Osborne T H et al 2000a Plasma Phys. Control. Fusion 42 A175

Osborne T H et al 2000b J. Nucl. Mater. 290-293 1013

Ozeki T et al 1990 Nucl. Fusion 301425

Parail V V et al 2001 Predictive modelling of JET plasmas with edge and core transport barriers. Proc. 28th Conf. Control. Fusion Plasma Phys. (Madeira) Europhys. Conf. Abstracts 25A 1873

Ryter F et al 2001 Nucl. Fusion 41537 
Saibene G et al 1998 High density ELMy H-modes studies at JET in ITER relevant scenarios Proc. 1998 Int. Congress on Plasma Phys. and 25th European Physical Society Conf. on Controlled Fusion and Plasma Physics Europhys. Conf. Abstracts 22C 0341

Saibene G et al 1999 Nucl. Fusion 391133

Saibene G et al 2001 The effect of plasma shape on density and confinement of ELMy H-Modes in JET Proc 28th Conf. on Controlled Fusion and Plasma Physics (Madeira) Europhys. Conf. Abstracts 25A 1749

Sartori R et al 2001a Studies of Type III ELMs in JET in preparation

Sartori R et al 2001b Edge operational space for high density-high confinement ELMy H-Modes in JET Proc 28th Conf. on Controlled Fusion and Plasma Physics (Madeira) Europhys. Conf. Abstracts 25A 937

Sartori R et al 2002 Edge operational space for high density-high confinement ELMy H-Modes in JET Plasma Phys. Control. Fusion 441801

Sips A C C et al 2002 Plasma Phys. Control. Fusion 44 A151

Snipes J A et al 2001 Plasma Phys. Control. Fusion 43 L23

Stober J et al 2000 Plasma Phys. Control. Fusion 42 A211

Stober J et al 2001a Nucl. Fusion 411123

Stober J et al 2001b Plasma Phys. Control. Fusion 43 A39

Valovic M et al 2002 Plasma Phys. Control. Fusion 441911

Wilson H R, Snyder P B and Huysmans G T A 2001 Coupled peeling-ballooning modes: a model for ELMs and the temperature pedestal? Proc. 28th Conf. on Controlled Fusion and Plasma Physics (Madeira) Europhys. Conf. Abstracts 25A 1753 